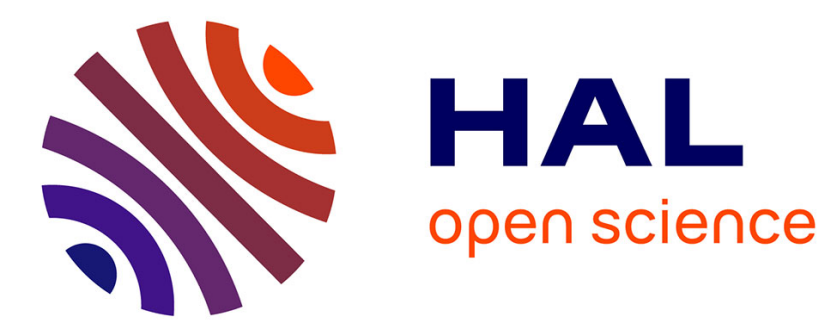

\title{
The TVL1 Model: a Geometric Point of View
}

Vincent Duval, Jean-François Aujol, Yann Gousseau

\section{To cite this version:}

Vincent Duval, Jean-François Aujol, Yann Gousseau. The TVL1 Model: a Geometric Point of View. Multiscale Modeling and Simulation: A SIAM Interdisciplinary Journal, 2009, 10.1137/090757083. hal-00380195

\section{HAL Id: hal-00380195 https://hal.science/hal-00380195}

Submitted on 30 Apr 2009

HAL is a multi-disciplinary open access archive for the deposit and dissemination of scientific research documents, whether they are published or not. The documents may come from teaching and research institutions in France or abroad, or from public or private research centers.
L'archive ouverte pluridisciplinaire HAL, est destinée au dépôt et à la diffusion de documents scientifiques de niveau recherche, publiés ou non, émanant des établissements d'enseignement et de recherche français ou étrangers, des laboratoires publics ou privés. 


\title{
THE TVL1 MODEL: A GEOMETRIC POINT OF VIEW
}

\author{
VINCENT DUVAL ${ }^{\dagger}$, JEAN-FRANÇOIS AUJOL ${ }^{\ddagger}$, AND YANN GOUSSEAU †
}

\begin{abstract}
.
The aim of this paper is to investigate the geometrical behavior of the TVL1 model used in image processing, by making use of the notion of Cheeger sets. This mathematical concept was recently related to the celebrated Rudin-Osher-Fatemi image restoration model, yielding important advances in both fields. We provide the reader with a geometrical characterization of the TVL1 model. We show that, in the convex case, exact solutions of the TVL1 problem are given by an opening followed by a simple test over the ratio perimeter/area. Shapes remain or suddenly vanish depending on this test. As a result of our theoritical study, we suggest a new and efficient numerical scheme to apply the model to digital images. As a by-product, we justify the use of the TVL1 model for image decomposition, by establishing a connection between the model and morphological granulometry. Eventually, we propose an extension of TVL1 into an adaptive framework, in which we derive some theoretical results.
\end{abstract}

Key-words. Total variation, Cheeger sets, mathematical morphology, adaptive image denoising

1. Introduction. The aim of this paper is to analyze the geometrical behavior of the TVL1 denoising model. This analysis is made possible by recent progresses in the study of Cheeger sets. Let us first recall the definition of this model. Consider a function $f: \mathbb{R}^{2} \rightarrow \mathbb{R}$. We are interested in finding some $u$ in $B V$, the space of functions with bounded variations, which solves the following minimization problem:

$$
\inf _{u \in B V\left(\mathbb{R}^{2}\right)}\left\{\int_{\mathbb{R}^{2}}|D u|+\lambda \int_{\mathbb{R}^{2}}|f-u| d x\right\}
$$

This type of energy was introduced by S. Alliney in $[4,3]$ in a discrete setting for one-dimensional signals. M. Nikolova showed in [35] that such an approach is efficient to restore images corrupted by impulse noise. Chan and Esedoglu then gave a first theoretical analysis of the model in [25], exhibiting some very specific properties. For instance, in the case when $f$ is the characteristic function of a disc, the solution of (1.1) is either $f$ itself or 0 , depending on the value of $\lambda$. Thanks to the notion of Cheeger sets, we will generalize this type of results in the present paper. Notice that this behavior is specific to the TVL1 model. For instance, in the celebrated TVL2 model (or Rudin-Osher-Fatemi, ROF, model) [38], one solves the problem:

$$
\inf _{u \in B V\left(\mathbb{R}^{2}\right)}\left\{\int_{\mathbb{R}^{2}}|D u|+\lambda \int_{\mathbb{R}^{2}}|f-u|^{2} d x\right\}
$$

It is well-known that if $f$ is the characteristic function of a disc, then the solution $u$ of (1.2) is also the characteristic function of the same disc, but with a smaller contrast, whatever the value of $\lambda$ in $(0,+\infty)$. This particular behavior of the solutions of the TVL1 model (1.1) was the motivation of the work in [25], that has

\footnotetext{
${ }^{\dagger}$ Institut Telecom, Telecom ParisTech, CNRS UMR 5141 ([vincent.duval, yann.gousseau]@telecom-paristech.fr)

${ }^{\ddagger}$ CMLA, ENS Cachan, CNRS, UniverSud (Jean-Francois.Aujol@cmla.ens-cachan.fr)
} 
been the inspiration of several subsequent works. Let us mention the work by Yin et al [44], where the connection of TVL1 with a geometric problem on level sets is investigated. We will emphasize on this connection in Section 4. Let us also mention the work by Allard $[2,1]$ which we noticed while writing this paper: as in the present paper, he also uses geometrical measure theory results to study the TVL1 model. In particular, regularity results similar to those of Section 5.2 are derived in a more general setting. The specificity of our analysis, apart from aiming explicitly at image processing applications, is that it is based on recent results by V. Caselles and his collaborators $[18,6,24]$. In particular, by focusing on the case of dimension 2 , we give a characterization of the solution of TVL1 in the convex case through classical operators from mathematical morphology. Moreover, it motivates an efficient geometrical scheme for minimizing the TVL1 model.

The plan of the paper is the following. In Section 2 and 3, we introduce some basic tools that will be used in the rest of the paper. In Section 4, we study the link between problem (1.1) and a geometrical problem on level sets. Section 5 is then devoted to the analysis of this geometrical problem. This is the most important section of the paper. In particular, we completely characterize the behavior of the solutions of (1.1) in the case when $f$ is the characteristic function of some convex set (Theorem 5.14). Thanks to this analysis, we can propose an efficient geometrical numerical scheme in Section 6. We illustrate this scheme with examples on images and we compare our results with a graph-cut algorithm introduced by Darbon and Sigelle in [27]. Eventually, we generalize our study in Section 7 to the case when the regularization parameter is no longer a constant.

\section{Notations and definitions.}

2.1. Functions of bounded variation. In this section, we recall some definitions and properties about functions of bounded variation. All considered functions will be defined on the plane and we shall denote by $L^{1}$ the space of integrable functions on $\mathbb{R}^{2}$. For more details, we refer the reader to [10] or [9].

Definition 2.1. A function $u \in L^{1}$ is said to be of bounded variation if its distributional gradient is a vector valued Radon measure with finite total variation. The total variation of $D u$ on an open set $\Omega \subset \mathbb{R}^{2}$ is equal to:

$$
|D u|(\Omega)=\sup \left\{\int_{\mathbb{R}^{2}} u \operatorname{div} \varphi / \varphi \in C_{c}^{1}\left(\Omega, \mathbb{R}^{2}\right), \forall x \in \Omega,|\varphi(x)| \leq 1\right\}
$$

(where for a vector $|v|=\left(v_{1}, v_{2}\right) \in \mathbb{R}^{2}$, we set $\left.|v|^{2}=v_{1}^{2}+v_{2}^{2}\right)$. When $\Omega=\mathbb{R}^{2}$, this quantity is called the total variation of $u$, and will be denoted by $|u|_{T V}$ (or sometimes $J(u)$ ). A\}.

The total variation of a Borel set $B \subset \mathbb{R}^{2}$ is defined as $\inf \{|D u|(A), A$ open,$B \subset$

If $u=\chi_{E}$ is the characteristic function of a measurable set $E \subset \mathbb{R}^{2}$ and has bounded variation, we say that $E$ is a set of finite perimeter, and write Per $E=$ $\left|D \chi_{E}\right|\left(\mathbb{R}^{2}\right)$, or Per $(E, \Omega)=\left|D \chi_{E}\right|(\Omega)$.

For sets of finite perimeter, one can define the reduced boundary $\partial^{*} E$ which is $\mathcal{H}^{1}$ countably rectifiable with finite one-dimensional Haussdorff measure $\mathcal{H}^{1}$ and compute the outer unit normal $\nu(x)$ at $\mathcal{H}^{1}$ almost every point $x \in \partial^{*} E$. Then $\left|D \chi_{E}\right|$ coincides with the restriction of $\mathcal{H}^{1}$ to $\partial^{*} E$.

If $E$ is of class $C^{1,1}$, the curvature of $\partial E$ (defined $\mathcal{H}^{1}$ almost everywhere) will be denoted by $\kappa$. 
Theorem 2.2 (Coarea Formula). Let $u \in B V$. Then:

$$
|D u|\left(\mathbb{R}^{2}\right)=\int_{-\infty}^{+\infty} \operatorname{Per}\left\{x \in \mathbb{R}^{2}, u(x)>t\right\} d t .
$$

For $u \in L^{1}$, if the right member of the equation is finite, then $u \in B V\left(\mathbb{R}^{2}\right)$ and the last equality holds.

In this paper, we only consider the case of dimension $N=2$. We thus have: $B V(\Omega) \subset L^{2}(\Omega)$ (with continuous embedding). We can then extend the total variation $|D u|$ as a convex lower semi-continuous function $J$ defined on $\mathbb{R}^{2}$ by:

$$
J(u)=\left\{\begin{array}{l}
|D u|(\Omega) \text { if } u \text { belongs to } B V(\Omega) \\
+\infty \text { if } u \in L^{2}(\Omega) \backslash B V(\Omega)
\end{array}\right.
$$

$J$ being a proper convex function, we can use the notion of subgradient $\partial J$ of $J$ [37]: $v \in \partial J(u)$ if and only if for all $w \in L^{2}(\Omega)$, we have $J(u+w) \geq J(u)+\langle v, w\rangle_{L^{2}(\Omega)}$ where $\langle., .\rangle_{L^{2}(\Omega)}$ stands for the usual $L^{2}(\Omega)$ inner product.

2.2. A generalized Green's formula. In order to recall the framework of [18],[6], [24], we need the following formula. Let $\Omega$ be an open subset of $\mathbb{R}^{2}$. Following [11], [12], define

$$
X_{\infty}(\Omega):=\left\{z \in L^{\infty}\left(\Omega, \mathbb{R}^{2}\right) / \operatorname{div} z \in L^{\infty}(\Omega)\right\} .
$$

If $z \in X_{\infty}(\Omega)$ and $w \in B V(\Omega)$ we define the functional $(z, D w): C_{0}^{\infty}(\Omega) \rightarrow \mathbb{R}$ by the formula:

$$
\langle(z, D w), \varphi\rangle:=-\int_{\Omega} w \varphi \operatorname{div} z d x-\int_{\Omega} w z \cdot \nabla \varphi d x .
$$

Then $(z, D w)$ is a Radon measure in $\Omega, \int_{\Omega}(z, D w)=\int_{\Omega} z \cdot \nabla w d x$ for all $w \in W^{1,1}(\Omega)$ and:

$$
\forall B \text { Borel set } \subset \Omega,\left|\int_{B}(z, D w)\right| \leq \int_{B}|(z, D w)| \leq\|z\|_{\infty} \int_{B}|D w| \text {. }
$$

TheOREM 2.3. Let $\Omega \subset \mathbb{R}^{2}$ be a bounded open set with Lipschitz boundary. Let $z \in X_{\infty}(\Omega)$. Then there exists a function $[z \cdot \nu] \in L^{\infty}(\partial \Omega)$ such that $\|[z \cdot \nu]\|_{L^{\infty}(\partial \Omega)} \leq$ $\|z\|_{L^{\infty}\left(\Omega, \mathbb{R}^{2}\right)}$, and for all $u \in B V(\Omega)$ :

$$
\int_{\Omega} u d i v z d x+\int_{\Omega}(z, D u)=\int_{\partial \Omega}[z \cdot \nu] u d \mathcal{H}^{1} .
$$

When $\Omega=\mathbb{R}^{2}$ we have the following integration by parts formula:

$$
\int_{\mathbb{R}^{2}} w \operatorname{div} z d x+\int_{\mathbb{R}^{2}}(z, D w)=0
$$


3. Some basic notions about calibrable and Cheeger sets. In [18], the authors characterize bounded sets with finite perimeter that evolve at constant speed by the total variation flow $\frac{\partial u}{\partial t}=\operatorname{div}\left(\frac{D u}{\mid D u}\right)$. Such sets $E \subset \mathbb{R}^{2}$ necessarily satisfy the condition $\lambda_{E} \chi_{E} \in \partial J\left(\chi_{E}\right)$ for some $\lambda_{E} \in \mathbb{R}$, where $\partial J$ is the subdifferential of the total variation. Those sets are called calibrable.

Definition 3.1. Let $E$ be a bounded set with finite perimeter. We say that $E$ is calibrable if there exists a vector field $\xi \in X_{\infty}$ with $\|\xi\|_{\infty} \leq 1$ such that - div $\xi=\lambda_{E} \chi_{E}$ in $\mathcal{D}^{\prime}\left(\mathbb{R}^{2}\right)$ for some $\lambda_{E}$, and $\left(\xi, D \chi_{E}\right)=\left|D \chi_{E}\right|$ as measures in $\mathbb{R}^{2}$.

In that case, $\lambda_{E}$ is uniquely determined by $\lambda_{E}=\frac{\operatorname{Per} E}{|E|}$ where $|E|$ denotes the two-dimensional (Lebesgue) area. In the rest of this paper, we shall use this notation to refer to $\frac{\operatorname{Per} E}{|E|}$ whether $E \subset \mathbb{R}^{2}$ is calibrable or not.

In this paper, we will not be directly using this definition but we will rather use the following two propositions ([18], [11]):

Proposition 3.2 ([18]). Let $F \subset \mathbb{R}^{2}$ be a calibrable set. Then:

(i)

$$
\frac{\text { Per } F}{|F|} \leq \frac{\text { Per } U}{|U \cap F|} \text { for all } U \subset \mathbb{R}^{2} .
$$

(ii) Each connected component of $F$ is convex.

Proposition 3.3. Let $C \subset \mathbb{R}^{2}$ be a bounded convex set with finite perimeter. The following assertions are equivalent:

(i) $C$ is calibrable

(ii) $C$ is a solution of the problem:

$$
\min _{X \subset C} \operatorname{Per} X-\lambda_{C}|X| \quad\left(\text { where } \lambda_{C}=\frac{\operatorname{Per} C}{|C|}\right) .
$$

A very interesting property of convex calibrable set is the following characterization, proved in ([29] Theorem A.1, [6], [18], [31] )

Proposition 3.4. Let $C$ be a bounded convex set. Then $C$ is calibrable iff the following two conditions hold:

(i) $\partial C$ is of class $C^{1,1}$

(ii) $\operatorname{ess}_{\sup } \operatorname{su}_{x \in C} \kappa(x) \leq \frac{\operatorname{Per} C}{|C|}$.

Example. It was proved in [31] that an ellipse with excentricity lower than $\bar{e} \approx$ 0.7192 is calibrable.

A related topic is the Cheeger problem. Given a nonempty open bounded set $F \subset \mathbb{R}^{2}$, find: $\lambda_{F}:=\inf _{X \subset F} \frac{\operatorname{Per} X}{|X|}$. Any minimizer $X$ of this problem is called a Cheeger set of $F$. If $F$ itself is a minimizer, we say that $F$ is Cheeger in itself.

Notice that $F$ is Cheeger in itself if and only if it is solution of the problem: $\min _{X \subset F}$ Per $X-\lambda_{F}|X|$. As a consequence, by Proposition 3.2, a calibrable set is Cheeger in itself, and the converse is true when the set is convex (by Proposition $3.3)$.

The following theorem is proved in [24] with the assumption that $C$ is uniformly convex with boundary of class $C^{2}$. In [5], these assumptions are removed.

TheOREM 3.5 ([24], [5]). Let $C$ be a non-trivial convex body (i.e. a nonempty compact convex subset of $\left.\mathbb{R}^{2}\right)$. Then there is a unique Cheeger set inside $C$. This set is convex and of class $C^{1,1}$.

This Cheeger set is given explicitely by the following theorem (see [7] and [31]): 
ThEOREM 3.6. Let $C$ be a non-trivial convex body, then there exists a unique value $t=t *>0$ such that the Cheeger set of $C$ is given by an opening with radius $t^{*}$. This value $t^{*}$ is the unique value of $t$ such that the area of the eroded set of $C$ with radius $t$ is equal to $\pi t^{2}$

\section{The geometric equivalence theorem and its consequences.}

4.1. The geometric equivalence. In this section, we first present a few basic results about the TVL1 functional, then we state the equivalence between TVL1 and a geometric problem.

For $f \in L^{1}$, we want to solve:

$$
\inf _{u \in L^{1}} \mathcal{E}(u):=|u|_{T V}+\lambda \int_{\mathbb{R}^{2}}|f(x)-u(x)| d x
$$

The following result is well-known: it is a standard application of the direct method of calculus of variations. We refer the reader to ([44], [25]) or to Section 7 for a proof in the more general case of adaptive TVL1.

Proposition 4.1. There exists at least one solution to the Problem (4.1). Let us define:

$$
T: L^{1} \rightarrow \mathcal{P}\left(L^{1}\right)
$$

the operator which maps $f$ to the set of solutions $T f=\left\{u \in L^{1}, u\right.$ is solution of (4.1) $\}$. The set $T f$ is a convex closed set in $L^{1}$.

Let us notice that, since the functional is not strictly convex, many solutions may exist. This is the reason why we need to define $T$ as a set-valued map.

It was noticed in [25] that, using the coarea formula, the energy $\mathcal{E}(u)$ could be reformulated as a sum of energies over the level sets of $u$ :

$$
\mathcal{E}(u)=\int_{-\infty}^{+\infty} \operatorname{Per}\{x, u(x)>\mu\}+\lambda|\{x, u(x)>\mu\} \triangle|\{x, f(x)>\mu\} \mid d \mu .
$$

In view of this formula, one may consider the family of geometric problems, given a function $f \in L^{1}$ :

$$
\inf _{U \subset \mathbb{R}^{2}} \operatorname{Per} U+\lambda\left|U \triangle F_{\mu}\right| \quad\left(P_{\mu}\right)
$$

where $\mu \in \mathbb{R}$ and $F_{\mu}=\{x, f(x)>\mu\}$.

Existence of solutions for each of these problems is standard, since it can be proved by the direct method of the calculus of variations. Here again, we can define a multivalued map $\mathcal{T}$ that maps $F_{\mu}$ to the set of solution sets $\mathcal{T} F_{\mu}$ for Problem $\left(P_{\mu}\right)$. Actually, in [25], the authors show that if the data is the characteristic function of a set $\left(f=\chi_{F}\right)$, then every level set of a solution $u$ of (4.1) is a solution of the geometric problem associated to $F$. In [44], it was pointed out that stacking solutions of the geometric problem lead to a solution of (4.1). The following theorem states the equivalence between the family of geometric problems on level sets and the functional TVL1 problem. Let us observe that this result is implicitly assumed in order to establish several properties in $[26,44]$, such as the contrast invariance of the TVL1 
model. It is however, to the best of our knowledge, not proved in the literature. Since it is not trivial, we propose the following formulation and proof.

THeOREM 4.2 (Geometric equivalence). Let $f \in L^{1}$. The following assertions are equivalent:

(i) $u$ is solution of (4.1)

(ii) Almost every level set $U_{\mu}$ of $u$ is a solution of (4.4).

We shall need the following monotonicity result. We state it in a similar way to Yin, Goldfarb and Osher [44]. Let us mention that it was also established in terms of Gibbs energy by Darbon and Sigelle [27] in order to derive their graph-cut algorithm. For a proof of this result, we refer the reader to ([44] Theorem 3.1, [27] Lemma 1). In Section 7.1, we will generalize this monotonicity result to the adaptive TVL1.

TheOREM 4.3 (Pseudo-monotonicity of the geometric problem). Let $S_{1} \subset S_{2} \subset$ $\mathbb{R}^{2}$, and $U_{i}, i=1,2$ be a solution the geometric problem associated to $S_{i}$. Then

- $U_{\wedge}=U_{1} \cap U_{2}$ is a solution associated to $S_{1}$

- $U_{\vee}=U_{1} \cup U_{2}$ is a solution associated to $S_{2}$

REMARK 4.4. Notice that it is equivalent to consider a function $f: \mathbb{R}^{2} \rightarrow \mathbb{R}$ or to consider a family of sets $\left(U_{\mu}\right)_{\mu \in \mathbb{R}}$ that have the nesting property:

$$
U_{\mu} \subset \bigcap_{\nu<\mu} U_{\nu}
$$

This fact is at the core of the following proof.

Proof. [of Theorem 4.2] Since the implication $(i i) \Rightarrow(i)$ is clear by the coarea formula, we only focus on proving $(i) \Rightarrow(i i)$.

Let us notice that the mapping $t \mapsto F_{t}$ is non-increasing for the inclusion, therefore it is continuous everywhere but on a countable set of points (we shall denote this set $\mathcal{J})$. Let us consider a countable dense set $D \subset \mathbb{R}$ (for instance $D=\mathbb{Q})$.

First step: We are going to construct by induction a family $\left(U_{q}\right)_{q \in D}$. To this end, let $\left(q_{n}\right)_{n \in \mathbb{N}}$ be an enumeration of $D$. We choose an optimal set $U_{q_{0}}$ for problem $\left(P_{q_{0}}\right)$. Let us assume that we have chosen $U_{q_{j}}(j=0 \ldots n)$ satisfying the nesting property, and let us consider $q_{n+1}$. Three cases are possible. If there exists $m, p \leq n$ such that $q_{m}<q_{n+1}<q_{p}$ (we can assume that $q_{m}$ (resp. $q_{p}$ ) is the greatest (resp. smallest) such element), then let us consider $U \subset \mathbb{R}^{2}$ solution of $\left(P_{q_{n+1}}\right)$, and set:

$$
U_{q_{n+1}}=\left(U \cap U_{q_{m}}\right) \cup U_{q_{p}}
$$

By induction hypothesis $U_{q_{p}} \subset U_{q_{n+1}} \subset U_{q_{m}}$, and therefore the family $\left.\left(U_{q_{j}}\right)_{j=0 \ldots n+1}\right)$ satisfies the nesting property. Theorem 4.3 ensures that $U_{q_{n+1}}$ is solution of $\left(P_{q_{n+1}}\right)$.

The other two cases $\left(\forall j \leq n, q_{j}<q_{n+1}\right.$ or $\left.\forall j \leq n, q_{j}>q_{n+1}\right)$ can be dealt with similarly (take $U \cap U_{q_{m}}$ or $U \cup U_{q_{p}}$ ).

Therefore we have built a countable family of optimal sets satisfying the nesting property.

Second step: Now let us define the family $\left(U_{t}^{*}\right)_{t \in \mathbb{R}}$ by:

$$
U_{t}^{*}=\bigcap_{q<t, q \in D} U_{q} .
$$

This family has the nesting property ; let us show that it is solution of problem $\left(P_{t}\right)$ for almost every $t \in \mathbb{R}$.

Let $t \in \mathbb{R} \backslash \mathcal{J}$, and $\left(t_{n}\right)$ an increasing sequence in $D$ converging to $t$. 
For all $V \subset \mathbb{R}^{2}$, Per $V+\lambda\left|V \Delta F_{t_{n}}\right| \geq$ Per $U_{t_{n}}+\lambda\left|U_{t_{n}} \Delta F_{t_{n}}\right|$. We take the lowerlimit as $n \rightarrow+\infty$ :

$$
\text { Per } V+\lambda\left|V \Delta F_{t}\right| \geq \operatorname{Per} U_{t}^{*}+\lambda\left|U_{t}^{*} \Delta F_{t}\right|
$$

where we used the continuity of $t \mapsto\left|F_{t}\right|$, and the lower semicontinuity of the perimeter. Eventually, the elements of the family $\left(U_{t}^{*}\right)_{t \in \mathbb{R}}$ are solutions of $\left(P_{t}\right)$, except maybe for $t \in \mathcal{J}$.

Conclusion: We can then build a function $u^{*}$ by the formula:

$$
u(x)=\sup \left\{t \in \mathbb{R}, x \in U_{t}^{*}\right\} .
$$

Let then $v$ be a solution of problem (4.1), and let us call $V_{t}$ its level sets. By the coarea formula, it is clear that $u$ is a solution too, thus:

$$
\int_{-\infty}^{+\infty} \operatorname{Per} V_{t}+\lambda\left|V_{t} \triangle F_{t}\right| d t=\int_{-\infty}^{+\infty} \operatorname{Per} U_{t}^{*}+\lambda\left|U_{t}^{*} \triangle F_{t}\right| d t
$$

with the inequality for almost every $t$ :

$$
\text { Per } V_{t}+\lambda\left|V_{t} \triangle F_{t}\right| \geq \operatorname{Per} U_{t}^{*}+\lambda\left|U_{t}^{*} \triangle F_{t}\right| .
$$

We deduce that there is actually equality for almost every $t$.

In the rest of this section, we will state a few properties of the TVL1 model that are desirable when processing images. Some of them (Propositions 4.7, 4.9, 4.10,4.11) are well-known ([26], [44]).

\subsection{Maximum principle and monotonicity.}

Proposition 4.5 (Maximum principle). Let $M, m \in \mathbb{R}$ and assume $m \leq f \leq M$ almost everywhere. Then:

$$
\forall u \in T f, m \leq u \leq M \text { almost everywhere. }
$$

Proof. Let us assume by contradiction that $u \in T f$ with $u(x)>M$ on a set with non-zero Lebsegue measure. Then the truncated function $u_{M}=\min (u, M)$ has energy strictly lower than $u$. Indeed:

$\left|u_{M}\right|_{T V}+\lambda \int_{\mathbb{R}^{2}}\left|f-u_{M}\right|=\int_{-\infty}^{M} \operatorname{Per}\{x, u(x)>\mu\}+\lambda|\{x, u(x)>\mu\} \triangle|\{x, f(x)>\mu\} \mid d \mu$

whereas:

$$
\begin{array}{r}
|u|_{T V}+\lambda \int_{\mathbb{R}^{2}}|f-u|=\int_{-\infty}^{M} \operatorname{Per}\{x, u(x)>\mu\}+\lambda|\{x, u(x)>\mu\} \triangle\{x, f(x)>\mu\}| d \mu \\
+\underbrace{\int_{M}^{+\infty} \operatorname{Per}\{x, u(x)>\mu\} d \mu}_{\geq 0}+\underbrace{\int_{M}^{+\infty} \lambda|\{x, u(x)>\mu\}| d \mu}_{=\lambda \int_{\mathbb{R}^{2}}(u(x)-M)+d x>0}
\end{array}
$$


which contradicts the fact that $u$ is a minimizer.

Because of the non-uniqueness of the solution, we have to precise the notion of monotonicity we will be using.

Definition 4.6. We say that an operator $\mathcal{A}: L^{1} \mapsto \mathcal{P}\left(L^{1}\right)$ is pseudo-monotone if, given $f, g \in L^{1}$ with $f \leq g$ a.e., we have:

$$
\forall u_{1} \in \mathcal{A} f, \forall u_{2} \in \mathcal{A} g, \max \left(u_{1}, u_{2}\right) \in \mathcal{A} g \text { and } \min \left(u_{1}, u_{2}\right) \in \mathcal{A} f
$$

A straightforward consequence of Theorems 4.2 and 4.3 is the following result:

Proposition 4.7 (Pseudo-monotonicity). $T$ is pseudo-monotone.

Yet, it can be handful to define the notion of "greatest" and "lowest " solutions in order to take advantage of the classical notion of monotonicity.

Definition 4.8. Let us define the operators $T^{+}$and $T^{-}: L^{1} \rightarrow L^{1}$ by:

$$
\begin{aligned}
& T^{-} f=\arg \min \left\{\int_{\mathbb{R}^{2}} u, u \in T f\right\} \\
& T^{+} f=\arg \max \left\{\int_{\mathbb{R}^{2}} u, u \in T f\right\}
\end{aligned}
$$

Each operator defines a unique function, and is monotone. Moreover, this function is a solution of (4.1).

Proof. Let us denote by $K$ the minimal value of the energy (4.1). We shall prove the result for $T^{-}$(the result for $T^{+}$will follow since $T^{+} f=-T^{-}(-f)$ ).

The infimum of $\left\{\int_{R^{2}} u, u \in T f\right\}$ is finite since:

$$
\forall u \in T f, \int_{\mathbb{R}^{2}}(u-f) \geq-\left(|u|_{T V}+\lambda \int_{\mathbb{R}^{2}}|f(x)-u(x)| d x\right)=-K .
$$

Now let $u_{n}$ be a minimizing sequence. By Proposition 4.7, we can always assume,by replacing $u_{n+1}$ by $\min \left(u_{n+1}, u_{n}\right)$, that the sequence $u_{n}(x)$ is non-increasing for almost every $x \in \mathbb{R}^{2}$.

Let $u^{*}(x)$ be the pointwise limit of $u_{n}(x)$. By Beppo-Levi's theorem (monotone convergence) we have that $u_{n}$ converges to $u^{*}$ in $L^{1}$, and we have:

$$
\int_{\mathbb{R}^{2}} u^{*}=\inf \left\{\int_{\mathbb{R}^{2}} u, u \in T f\right\} .
$$

Since the set of solutions of (4.1) is closed in $L^{1}, u^{*}$ is a solution.

Now, we need to prove that $u^{*}$ does not depend on the choice of the sequence $u_{n}$. Let $v_{n}$ be another minimizing sequence, then the associated limit $v^{*}$ is a solution of (4.1). By Proposition 4.7, $\min \left(u^{*}, v^{*}\right)$ is a solution too, and its integral is lower than those of $u^{*}$ et $v^{*}$. This is only possible if $u(x)=v(x)$ almost everywhere.

Eventually, monotonicity is a consequence of Proposition 4.7.

Let us notice that $T^{-} f$ actually defines the lowest solution, and $T^{+} f$ the greatest. Problem (4.1) has a unique solution iff $T^{-} f=T^{+} f$.

In a similar way, we can define the notion of largest and smallest solution to the geometric problem, and denote them by $\mathcal{T}^{+}, \mathcal{T}^{-}$. 
4.3. Commutation with constants and affine invariance. In this subsection and only in this subsection, we shall consider data functions $f \in L^{1}+C$, that is, sums of integrable functions and constants. Indeed, it is straightforward to extend the problem to such functions since the total variation is not affected by the addition of constants. The proofs of the next two propositions are elementary and omitted for brevety.

Proposition 4.9. The operator $T$ commutes with the addition of constants:

$$
\forall f \in L^{1}, \forall C \in \mathbb{R}, T(f+C)=T(f)+C,
$$

and is self-dual:

$$
\forall f \in L^{1}, T(-f)=-T(f) .
$$

Proposition 4.10. The operator $T$ commutes with translations and rotations:

$$
\forall f \in L^{1}, \forall R \in S O_{n}, T(f \circ R)=T(f) \circ R
$$

4.4. Contrast Invariance. As observed in [26, 44], the following result is a direct consequence of the geometric equivalence Theorem 4.2.

Proposition 4.11 (Contrast invariance). Let $g$ be an increasing $C^{1}$-diffeomorphism with $\sup _{\mathbb{R}}\left|g^{\prime}\right|<+\infty$. Then: $T(g \circ f)=g(T f)$.

Notice that one usually uses contrast invariance of a functional operator to derive the existence of a level set formulation [30]. Interestingly enough, in the case of TVL1 we had to take the converse way.

5. The geometric problem. As it was shown in the last section, the study of the TVL1 problem amounts to solving the following geometric problem:

$$
\inf _{U \subset \mathbb{R}^{2}} \mathcal{E}_{G}(U):=\operatorname{Per} U+\lambda|U \triangle F|
$$

where $F \subset \mathbb{R}^{2}$ is a measurable set (in fact it is a given level set of the data).

5.1. Suddenly vanishing sets. An interesting property of the TVL1 model is the fact that some sets suddenly vanish. For instance, in [25], it is proved that the model preserves characteristic functions of discs with radius $R$ if $\lambda>2 / R$; below this value, the solution is the null function. In this subsection we focus on sets which have the same property. Those sets are necessarily Cheeger in themselves.

Proposition 5.1. Let $F \subset \mathbb{R}^{2}$ be a non empty set (not necessarily convex). Let us assume that $F$ has the thresholding property, i.e. there is some critical value $\tilde{\lambda}>0$ such that:

- $F$ is a solution of the geometric problem (5.1) for $\lambda>\tilde{\lambda}$.

- $\emptyset$ is a solution of the geometric problem (5.1)for $0<\lambda<\tilde{\lambda}$.

Then $F$ is Cheeger in itself, and $\tilde{\lambda}=\lambda_{F}:=\frac{\text { Per } F}{|F|}$.

Proof. Let us first notice that $\emptyset$ and $F$ are solutions of the geometric problem for $\lambda=\tilde{\lambda}$ (consider a monotone sequence $\left(\lambda_{n}\right)_{n \in \mathbb{N}}$ converging to $\tilde{\lambda}$ and pass to the limit in the inequality). Therefore, both sets have the same energy: Per $\emptyset+\tilde{\lambda}|F|=\operatorname{Per} F-0$. and we deduce that $\tilde{\lambda}=\frac{\text { Per } F}{|F|}=\lambda_{F}$.

Then, considering only sets $U$ that are included in $F$, we notice that $F$ is a non empty solution of the problem: $\inf _{U \subset F} \operatorname{Per} U-\lambda_{F}|U|$, which means that $F$ is Cheeger in itself. 
The converse is true in the convex case (in which case Cheeger in itself is equivalent to calibrable), but in general, not every Cheeger set in itself has the thresholding property. Here is a sufficient condition though:

Proposition 5.2. Let $F \subset \mathbb{R}^{2}$ be a calibrable set. Then $F$ has the thresholding property. More precisely:

- $F$ is the unique solution of the geometric problem (5.1) for $\lambda>\lambda_{F}$

- Both $\emptyset$ and $F$ are solutions for $\lambda=\lambda_{F}$

- $\emptyset$ is the unique solution for $\lambda<\lambda_{F}$

where $\lambda_{F}:=\frac{\operatorname{Per} F}{|F|}$.

Notice that, when $\lambda=\tilde{\lambda}$, other solutions than $F$ and $\emptyset$ may exist . For instance, consider the union of two discs $\left(F=D_{1} \cup D_{2}\right)$ with same radius $R$. Provided they are far enough from each other, they behave independantly (see Section 5.3.3) and for $\lambda=2 / R$ the solutions are $\emptyset, D_{1}, D_{2}$ and $D_{1} \cup D_{2}$.

Proof. Let us recall that, for all $V$ with finite perimeter, $\frac{\operatorname{Per} F}{|F|} \leq \frac{\operatorname{Per} V}{|V \cap F|}$.

- For $\lambda<\lambda_{F}$, we have:

$$
\begin{aligned}
\mathcal{E}_{G}(V) & =\operatorname{Per} V-\lambda|V \cap F|+\lambda|V \cup F| \\
& =\underbrace{\operatorname{Per} V-\lambda_{F}|V \cap F|}_{\geq 0}+\underbrace{\left(\lambda_{F}-\lambda\right)}_{>0}|V \cap F|+\lambda|V \cup F| \\
& \geq \lambda|F|=E(\emptyset)
\end{aligned}
$$

and this inequality is strict if $|V \cap F| \neq 0$. But, if $|V \cap F|=0$, then this quantitiy is strictly minimized when $|V|=0$.

- For $\lambda=\lambda_{F}$, let us write:

$$
\begin{aligned}
\mathcal{E}_{G}(V) & =\underbrace{\operatorname{Per} V-\frac{\operatorname{Per} F}{|F|}|V \cap F|}_{\geq 0}+\frac{\operatorname{Per} F}{|F|}|V \cup F| \\
& \geq \operatorname{Per} F \frac{|F \cup V|}{|F|} \\
& \geq \operatorname{Per} F=\mathcal{E}_{G}(F) .
\end{aligned}
$$

Therefore $F$ is a solution.

Moreover $\emptyset$ is a solution (passing to the limit with a sequence $\lambda_{n} \nearrow \lambda_{F}$ ).

- For $\lambda>\lambda_{F}=\frac{\text { Per } F}{|F|}$ :

$$
\begin{aligned}
\mathcal{E}_{G}(V) & =\underbrace{\operatorname{Per} V+\lambda_{F}|V \triangle F|}_{\geq \operatorname{Per} F}+\left(\lambda-\lambda_{F}\right)|V \triangle F| \\
& \geq \operatorname{Per} F+\underbrace{\left(\lambda-\lambda_{F}\right)}_{>0}|V \triangle F| \\
& \geq \mathcal{E}_{G}(F)
\end{aligned}
$$

and this inequality is strict if $V \neq F$.

\section{口}

As a conclusion, every calibrable set suddenly vanishes by TVL1, and every suddenly vanishing set is Cheeger in itself. The converse implications are false. In [44], it is shown that an annulus with large enough inner radius suddenly vanishes. It 

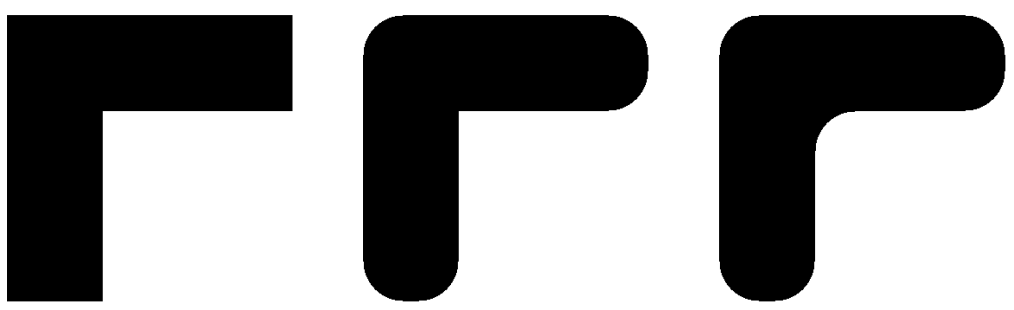

FIG. 5.1. The property Cheeger in itself imposes regularity "from the inside" (an upper bound on the curvature) whereas the property of being invariant by TVL1 requires regularity from "both sides" (the curvature is lower and upper-bounded). Therefore some Cheeger sets do not have the suddenly vanishing property. From left to right: a set with corners, its cheeger set, the solution of TVL1 with same curvature.

is connected and not convex, therefore it is not calibrable. Moreover, since suddenly vanishing sets are solutions of TVL1, they are generally smoother than Cheeger sets in themselves (see Figure 5.1).

\subsection{Smoothness of the boundary.}

REMARK 5.3. As noticed by Alliney [4], if $u$ is a solution of the TVL1 problem associated to $f$, then $u$ is a solution to the TVL1 problem associated to itself, since:

$$
|v|_{T V}+\lambda \int|u-v| \geq|v|_{T V}+\lambda \int(|f-v|-|f-u|) \geq|u|_{T V} .
$$

Or equivalently: TVL1 is idempotent. As a consequence, it is sufficient to study the sets that are invariant by TVL1 in order to describe all the sets that are solutions of the geometric problem for some data.

Proposition 5.4. Let $F \subset \mathbb{R}^{2}$, and $U \subset \mathbb{R}^{2}$ be a solution of the TVL1 problem associated to $F$. Then $\partial U$ coincides with the reduced boundary $\partial^{*} U$ of $U$ in the sense that $\mathcal{H}^{1}\left(\partial U \backslash \partial^{*} U\right)=0$, and the later is a $C^{1,1}$ hypersurface.

Proof. We rely on results from [9]. Let us notice that $U$ is a strong $\lambda$-minimizer ([9], Definition 4.7.3) in $\mathbb{R}^{2}$, that is:

$$
\operatorname{Per}(U, \Omega) \leq \operatorname{Per}\left(U^{\prime}, \Omega\right)+\lambda\left|U \triangle U^{\prime}\right|
$$

for every open set $\Omega$ and every $U^{\prime}$ with locally finite perimeter such that $U \triangle U^{\prime} \subset \subset \Omega$.

Indeed, let $\Omega$ and $U^{\prime}$ be such sets: then $\left|D \chi_{U}\right|\left(\mathbb{R}^{2} \backslash \Omega\right)=\left|D \chi_{U^{\prime}}\right|\left(\mathbb{R}^{2} \backslash \Omega\right)$. By the above remark, $U$ is a solution of TVL1 associated to $U$. Therefore:

$$
\operatorname{Per}(U, \Omega) \leq \operatorname{Per}\left(U^{\prime}, \Omega\right)+\lambda\left|U \triangle U^{\prime}\right|
$$

By corollary 4.2.4 in [9], $\mathcal{H}^{1}\left(\partial U \backslash \partial^{*} U\right)=0$, and the result stems from Theorems 4.7.1 and 4.7.4 in [9].

The next proposition shows that at points where the boundary of the solution associated to $F$ does not coincide with the boundary of $F$, the curvature is equal to $\pm \lambda$.

Proposition 5.5. Let $F \subset \mathbb{R}^{2}, U \subset \mathbb{R}^{2}$ a solution of the geometric problem (5.1) associated to $F, x \in \partial U, \kappa(x)$ the curvature of $\partial U$ at $x$, and $V$ a neighborhood of $x$. 
- If $V \cap F=\emptyset$ then $\kappa(x)=\lambda$

- If $V \cap F=V$ then $\kappa(x)=-\lambda$.

Notice that, if no such $V$ can be found, then $\partial F$ and $\partial U$ coincide at $x$. The idea is that, restricted to $V$, the geometric problem is equivalent to the famous mean curvature problem [9].

Proof. Without loss of generality, and up to a rotation of the axes, we can assume that $V=D \times I$ where $D \subset \mathbb{R}$ and $I \subset \mathbb{R}$ are bounded open intervalls and $V \cap U$ is the hypograph of a $C^{1,1}$ function $\phi: D \rightarrow I$.

The geometric energy classicaly reformulates in case (i):

$$
\mathcal{E}_{G}(U)=C+\int_{D} \sqrt{1+|\nabla \phi(x)|^{2}} d x+\lambda \int_{D} \phi(x) d x
$$

and in case (ii):

$$
\mathcal{E}_{G}(U)=C+\int_{D} \sqrt{1+|\nabla \phi(x)|^{2}} d x+\lambda \int_{D}(\sup I-\phi(x)) d x
$$

where $C$ is the energy outside $V$.

This is the prescribed mean curvature problem and the result follows by ([9], Theorem 1.1.3). For the convenience of the reader, we recall the proof, working with case (i). We consider a small perturbation of $\phi:(\phi+\epsilon \varphi)$ where $\varphi \in C_{c}^{1}(D)$. Then the energy becomes:

$$
\int_{D} \sqrt{1+|\nabla \phi(x)+\epsilon \nabla \varphi|^{2}} d x+\lambda \int_{D}(\phi+\epsilon \varphi(x)) d x
$$

and since it is minimal in $\epsilon=0$, its derivative vanishes:

$$
\forall \varphi \in C_{c}^{1}(D), \quad \int_{D} \frac{\langle\nabla \phi, \nabla \varphi\rangle}{\sqrt{1+|\nabla \phi|^{2}}}+\lambda=0
$$

which means:

$$
\operatorname{div}\left(\frac{\nabla \phi}{\sqrt{1+|\nabla \phi|^{2}}}\right)=\lambda \text {. }
$$

The left term is exactly $\kappa(x)$.

Remark. Using similar techniques one can prove that in fact:

$$
\text { ess } \sup _{x \in \partial U}|\kappa(x)| \leq \lambda
$$

5.3. Reformulation of the energy for a convex data. To begin with, let us point out that if $F$ is convex, the energy can be simplified.

Proposition 5.6. Let us assume that $F \subset \mathbb{R}^{2}$ is convex. Then, the geometric problem (5.1) associated to $F$ reformulates:

$$
\inf _{U \subset F} \operatorname{Per} U-\lambda|U| \text {. }
$$

Proof. The idea is to remark that every solution $U$ of the geometric problem (5.1) is contained in $F$ (modulo a Lebesgue negligible set). 
Indeed, let $U \subset \mathbb{R}^{2}$. By contradiction, if $|U \backslash F|>0$, let us prove that the geometric energy $\mathcal{E}_{G}(U \cap F)$ is strictly lower than $\mathcal{E}_{G}(U)$. Since $F$ is convex ${ }^{1}$ : Per $(U \cap$ $F) \leq \operatorname{Per} U$ (see [8]). We add $\lambda|F \backslash U|$ on both sides, and we get:

$$
\text { Per }(U \cap F)+\lambda|F \backslash U|<\text { Per } U+\lambda(|F \backslash U|+|U \backslash F|) \text { since }|U \backslash F|>0 \text {. }
$$

Which precisely means $\mathcal{E}_{G}(U \cap F)<\mathcal{E}_{G}(U)$, and contradicts the fact that $U$ is a solution. Thus, the geometric energy reformulates:

$$
\begin{aligned}
\operatorname{Per} U+\lambda|U \triangle F| & =\operatorname{Per} U+\lambda(|U|+|F|-2|U \cap F| \\
& =\operatorname{Per} U-\lambda|U|+\lambda|F| .
\end{aligned}
$$

The last term is constant. It has no influence on the choice of the solution.

Let us also recall a monotonicity result regarding the fidelity parameter $\lambda$. The following proposition can be found in ([6], Lemma 4) or [16]:

Proposition 5.7 (Monotonicity with $\lambda$ ). Let $0<\lambda<\mu$ and $U^{\lambda}, U^{\mu}$ be solutions of Problem (5.11) with fidelity parameter $\lambda$ (resp. $\mu$ ). Then $U^{\lambda} \subset U^{\mu}$.

In the convex case, calibrable is equivalent to Cheeger in itself. Therefore we can state the following proposition (uniqueness is provided by Theorem 3.5).

Proposition 5.8 (Suddenly vanishing convex sets). Let $F \subset \mathbb{R}^{2}$ be a convex set. Then $F$ has the thresholding property if and only if it is calibrable. Moreover, when $\lambda=\lambda_{F},\{\emptyset, F\}$ is exactly the set of solutions.

5.3.1. Convex sets that are invariant by TVL1. In view of Propositions 5.5 and 5.6, it is very easy to see that, given any convex set that is invariant by TVL1, both its curvature and its ratio perimeter/area are less than $\lambda$. A very interesting property is that the converse is true, as proved in [6]. Indeed, Problem (5.11) was studied in [6] in dimension $N$, in order to characterize convex calibrable sets. While relying on some of their results (notably the next theorem), we shall follow a path which is specific to dimension 2, in order to draw a parallel between the TVL1 model and mathematical morphology.

TheOrem 5.9 ([6], Theorem 9). Let $C$ be a convex $C^{1,1}$ bounded set of $\mathbb{R}^{2}$ and, as before, $\lambda_{C}=\operatorname{Per} C /|C|$. Let $\Lambda=\operatorname{ess} \sup _{x \in \partial C}|\kappa(x)|$. Then $C$ is a solution of the geometric problem (5.11) associated to $C$ (with fidelity parameter $\lambda$ ) if and only if $\max \left(\lambda_{C}, \Lambda\right) \leq \lambda$.

In view of Remark 5.3, let us notice that this theorem describes all convex solutions of the TVL1 problem: a convex set $C$ is solution of TVL1 for some $F$ iff $\max \left(\lambda_{C}, \Lambda\right) \leq \lambda$. Notice that this criterion combines both a local and a global term.

5.3.2. Characterization of solutions for a convex data. In this subsection, we explain how one can obtain the exact solution of the geometric problem using openings and a simple thresholding procedure. By the geometric equivalence Theorem 4.2 , this gives the exact solution of the TVL1 problem in case the data $f$ is convex or quasi-convex (i.e. its level sets are convex).

Definition 5.10. Let $C \subset \mathbb{R}^{2}$. Let us define the erosion and the opening with radius $r>0$ of $C$ by:

$$
C_{r}:=C \ominus B(0, r):=\{x \in C, B(x, r) \subset C\}
$$

\footnotetext{
${ }^{1} \mathrm{~A}$ similar argument ensures that even in the non convex case, solutions of TVL1 are contained in the convex hull of $F$.
} 


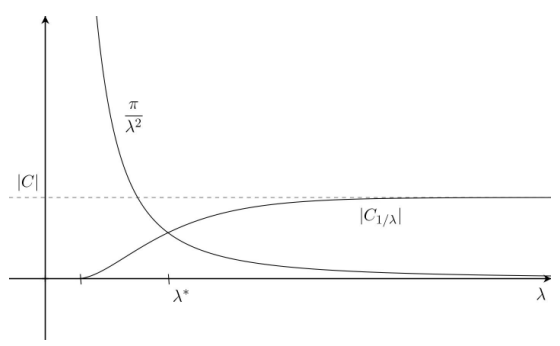

FIG. 5.2. General profile of the function $\lambda \mapsto\left|C_{1 / \lambda}\right|$ for a convex $C$. The value $\lambda^{*}$, at the intersection with the graph of the function $\pi / \lambda^{2}$, is such that the opening $C^{1 / \lambda^{*}}$ of $C$ is the unique Cheeger set of $C$.

$$
C^{r}:=C_{r} \oplus B(0, r)=\bigcup_{B(x, r) \in C} B(x, r)
$$

The following result can be found in [11], and is a consequence of Lemma 9.2 in [19].

Lemma 5.11 ([11] Lemma 4.27). Let $C \subset \mathbb{R}^{2}$ be an open convex bounded set. The following assertions are equivalent:

- There exists some $\rho>0$ such that $C=C^{\rho}$.

- $\partial C$ is of class $C^{1,1}$, and $\sup _{p \in \partial C} \operatorname{ess} \kappa_{\partial C}(p) \leq \frac{1}{\rho}$.

Next proposition describes the evolution of the ratio perimeter/area when performing openings.

Proposition 5.12 ([7], [31]). The mapping $\lambda \mapsto\left|C_{\frac{1}{\lambda}}\right|$ is continuous, increasing on $\mathbb{R}_{+}^{*}$, vanishes in a neighborhood of 0 and tends to $|C|$ in $+\infty$. Moreover:

$$
\frac{\operatorname{Per} C^{1 / \lambda}}{\left|C^{1 / \lambda}\right|}<\lambda \Leftrightarrow\left|C_{1 / \lambda}\right|<\frac{\pi}{\lambda^{2}} .
$$

Eventually, there exists a unique $\lambda^{*}$ such that $\frac{\operatorname{Per} C^{1 / \lambda^{*}}}{\left|C^{1 / \lambda^{*}}\right|}=\lambda^{*}$ (which is equivalent to $\left.\left|C_{1 / \lambda^{*}}\right|=\frac{\pi}{\lambda^{* 2}}\right)$.

Notice that the value $\lambda^{*}$ is precisely the one for which the opening $C^{1 / \lambda^{*}}$ is the Cheeger set of a convex body $C$ (see Section 3 ).

Proof. For the continuity of the mapping, we refer the reader to [7]. We only show here why the term $\frac{\pi}{\lambda^{* 2}}$ appears. By Steiner's formulas:

$$
\begin{gathered}
\forall r>0, \text { Per }\left(C_{r} \oplus B(0, r)\right)=\text { Per } C_{r}+2 \pi r \\
\left|C_{r} \oplus B(0, r)\right|=\left|C_{r}\right|+r \operatorname{Per} C_{r}+\pi r^{2} .
\end{gathered}
$$

Therefore:

$$
\frac{\operatorname{Per}\left(C_{r} \oplus B(0, r)\right)}{\left|C_{r} \oplus B(0, r)\right|}>\frac{1}{r} \Leftrightarrow r \operatorname{Per} C_{r}+2 \pi r^{2}>\left|C_{r}\right|+r \text { Per } C_{r}+\pi r^{2} .
$$

Lemma 5.13. Let $C \subset \mathbb{R}^{2}$ be a convex set, and $\lambda>0$. The largest convex $C^{1,1}$ open set included in $C$ whose curvature is lower than $\lambda$ is $C^{1 / \lambda}$.

Proof. 
- Let $\tilde{C}$ be an open $C^{11}$ convex set contained in $C$ with curvature lower than $\lambda$. Then, by Lemma $5.11, \tilde{C}^{1 / \lambda}=\tilde{C}$.

But, by monotonicity of the opening: $\tilde{C}^{1 / \lambda} \subset C^{1 / \lambda}$. Thus: $\tilde{C} \subset C^{1 / \lambda}$.

- It is clear that $C^{1 / \lambda}$ is convex (since if $C$ contains two balls it contains their convex hull) and it is open. By Lemma 5.11, $C^{1 / \lambda}$ is $C^{1,1}$ and its curvature is lower than $\lambda$.

We are now in a position to state the main result of this section, giving the solutions of the binary TVL1 problem in the convex case by means of operators from mathematical morphology.

THEOREM 5.14 (Solutions of the convex problem). Let $C$ be a bounded, convex set, and $\lambda^{*}$ defined by Proposition (5.12) (i.e. such that $\left|C_{1 / \lambda^{*}}\right|=\frac{\pi}{\lambda^{* 2}}$ )

- For $\lambda>\lambda^{*}$, the set $C^{1 / \lambda}$ is the unique solution of the geometric problem (5.11).

- For $\lambda=\lambda^{*}$, the set of solutions is exacly $\left\{C^{1 / \lambda^{*}}, \emptyset\right\}$, and $C^{1 / \lambda^{*}}$ is the Cheeger set of $C$.

- For $\lambda<\lambda^{*}$ the unique solution of Problem (5.11) is $\emptyset$.

Proof.

For $\lambda>\lambda^{*}$, let us define $C^{*}=\mathcal{T}^{+} C$ (see Definition 4.8). Then $C^{*}$ is $C^{1,1}$ (Proposition 5.4) and its curvature is lower than $\lambda$ (Proposition 5.9).

$C^{1 / \lambda}$ is the greatest convex $C^{1,1}$ open set contained in $C$ with curvature lower than $\lambda$ (see Lemma 5.13), so that int $C^{*} \subset C^{1 / \lambda} \subset C$, and as a result:

$$
\mathcal{T}^{+}\left(\operatorname{int} C^{*}\right) \subset \mathcal{T}^{+} C^{1 / \lambda} \subset \mathcal{T}^{+} C^{*}
$$

But the extreme terms are equal to $C^{*}$ (because we can identify int $C^{*}$ and $C^{*}$, since the $C^{1,1}$ boundary has null measure), therefore modulo a Lebesgue negligible set , $\mathcal{T}^{+} C^{1 / \lambda}=C^{*}$.

Eventually, $C^{1 / \lambda}$ is invariant by TVL1 (since $\frac{\operatorname{Per} C^{1 / \lambda}}{\left|C^{1 / \lambda}\right|} \leq \lambda$ and $\kappa \leq \lambda$ ), hence:

$$
C^{1 / \lambda}=\mathcal{T}^{+} C^{1 / \lambda}=C^{*}
$$

and $C^{1 / \lambda}$ is solution of the geometric problem.

We still have to prove uniqueness. Let $U \subset C$ be a solution associated with the value $\lambda$. Let $\lambda_{n}$ (resp. $\mu_{n}$ ) be an increasing (resp. decreasing) sequence with limit $\lambda$. The sets $C^{1 / \lambda_{n}}$ are solutions of (5.11) associated to $\lambda_{n}$, thus, by monotonicity in $\lambda$ (Proposition (5.7)):

$$
\bigcup_{n} C^{1 / \lambda_{n}} \subset U \subset \bigcap_{n} C^{1 / \mu_{n}}
$$

Both extreme terms are equal to $C^{1 / \lambda}$. Hence uniqueness.

For $\lambda=\lambda^{*}$, we compare the energy to the Cheeger problem. If $|U|>0$ :

$$
\text { Per } U-\lambda^{*}|U|=|U|\left(\frac{\operatorname{Per} U}{|U|}-\lambda^{*}\right)>0
$$

except if $U=C^{1 / \lambda^{*}}$, in which case the energy is equal to 0 , as well as the energy of $\emptyset$. 
For $\lambda<\lambda^{*}$ :

$$
\begin{aligned}
\text { Per } U-\lambda|U| & =\text { Per } U-\lambda^{*}|U|+\left(\lambda^{*}-\lambda\right)|U| \\
& >0 \text { except if } U=\emptyset .
\end{aligned}
$$

At this point we can look back at the thresholding property of convex calibrable sets (Proposition 5.8). These sets suddenly disappear because when $\lambda>\lambda^{*}$ they are invariant to an opening with radius $1 / \lambda$ (since, by characterization of convex calibrable sets, $\left.\kappa(x) \leq \frac{\operatorname{Per} C}{|C|}=\lambda^{*}\right)$.

In the general convex case, the last shape we see when decreasing $\lambda$ is the Cheeger set of $C$. One should relate this with the Rudin-Osher-Fatemi model and the following result of [6], [23] which is the key to the study of Cheeger sets in dimension $N$. Given a data $f=\chi_{C}$, denote by $u$ the unique solution of this problem, then the set $\{x, u(x) \geq$ $\left.\|u\|_{\infty}\right\}$ is the Cheeger set of $C$. The similarity between the two problems stems from the fact that in the convex case, the ROF model behaves like a "stack of geometric problems": the level sets $\left(U_{t}\right)_{t \in[0,1]}$ minimize the quantity Per $U-\lambda(1-t)|U|$.

This explains why Theorem 5.9, which was proved in the study of Cheeger sets, ended up so helpful to the study of the TVL1 problem.

5.3.3. Separated convex components. We are now in a position to deal with the case of several convex connected components, provided they are far enough from one another.

Proposition 5.15. Let $C_{1}, \ldots, C^{m} \subset \mathbb{R}^{2}$, be non empty connex bounded sets such that $\bar{C}_{i} \cap \bar{C}_{j}=\emptyset$ for $i \neq j$, and $F=\bigcup_{i=1}^{m} C_{i}$. Assume the sets $C_{i}$ have the following property:

$$
\text { (C) } \forall U \supset F \text {, Per }(U) \geq \sum_{i=1}^{m} \operatorname{Per}\left(C_{i}\right) \text {. }
$$

Let us define a partition of every set $U \subset \mathbb{R}^{2}$ by $U=\bigcup_{i=0}^{m} U_{i}$ with $U_{0} \subset F^{C}$ and $U_{i} \subset C_{i}$. Then, the geometric problem (5.1) can be split into independant problems:

$$
\forall i=1, \ldots, m, \min _{U_{i}} \operatorname{Per} U_{i}+\lambda\left|C_{i} \backslash U_{i}\right|
$$

Condition $(\mathcal{C})$ intuitively means that the components $C_{i}$ are far enough one from another. It also implies that the $C_{i}$ 's are convex. We can therefore apply results from last subsection to characterize the solution in this case.

Proof.

Notice that by submodularity:

$$
\operatorname{Per}\left(U \cup \bigcup_{i=1}^{m} C_{i}\right)+\operatorname{Per}\left(U \cap \bigcup_{i=1}^{m} C_{i}\right) \leq \operatorname{Per} U+\underbrace{\operatorname{Per}\left(\bigcup_{i=1}^{m} C_{i}\right)}_{=\sum_{i=1}^{m} \operatorname{Per} C_{i}}
$$

(since the distance between the $C_{i}$ is strictly positive). But $\operatorname{Per}\left(U \cup \bigcup_{i=1}^{m} C_{i}\right) \geq$ $\sum_{i=1}^{m} \operatorname{Per} C_{i}$ by condition (C). Thus, the inequality can hold only if: Per $\left(U \cap \bigcup_{i=1}^{m} C_{i}\right) \leq$ 
Per $U$. Moreover:

$$
\left|\left(U \cap \bigcup_{i=1}^{m} C_{i}\right) \triangle F\right|=\sum_{i=1}^{m}\left|C_{i} \backslash U_{i}\right| \leq\left|U_{0}\right|+\sum_{i=1}^{m}\left|C_{i} \backslash U_{i}\right| \leq|U \triangle F| .
$$

We can infer that $\mathcal{E}_{G}\left(U \cap \bigcup_{i=1}^{m} C_{i}\right) \leq \mathcal{E}_{G}(U)$. The geometric problem (5.1) amounts then to: $\min \sum_{i=1}^{m}\left(\operatorname{Per} U_{i}+\lambda\left|C_{i} \backslash U_{i}\right|\right)$. These problems are independant because $U_{i} \subset C_{i}$ and the $C_{i}$ 's are distant from one another.

Notice that Condition $(\mathrm{C})$, which is here only a sufficient condition, is very similar to conditions that appear naturally when studying calibrable sets (see [18], Theorem $6)$.

REMARK 5.16. Although Condition $(C)$ may not be optimal, notice that a condition on the distance between connected components appears necessary. This is easy to check in the particular case of two discs of radius $r$. Assume the distance between their centers is $d \geq 2 r$, then their convex hull has lower energy than the two discs when $2 r \leq d \leq \frac{\pi r}{2}\left(1+\frac{1}{1+\lambda r}\right)$. One can choose such $d$ as soon as $\lambda<\frac{1}{r}\left(\frac{2 \pi-4}{4-\pi}\right) \approx \frac{2.6}{r}$. For $\lambda \in\left(\frac{2}{r}, \frac{1}{r}\left(\frac{2 \pi-4}{4-\pi}\right)\right)$, the independant evolution predicts that the solution is two discs, which is contradicted by the fact that the convex hull has a lower energy.

\subsection{Mathematical morphology almost everywhere...}

5.4.1. TVL1 and mathematical morphology. In the convex case, we have seen that TVL1 is equivalent to an opening followed by a thresholding. When the connected components are non convex or too close to one another, things get more complicated and one may wonder if a simple morphological operator can describe or approximate the TVL1 model. K. R. Vixie and S. Esedoglu prove in [42] the following result by geometric arguments:

Proposition 5.17 ([42]). Let $F \subset \mathbb{R}^{2}$ be a bounded measurable set, and $U$ be a solution of the corresponding geometric problem (5.1). Assume that a ball $B\left(x, \frac{2}{\lambda}\right)$ lies completely in $F$. Then, $U \cup B\left(x, \frac{2}{\lambda}\right)$ is a solution of the geometric problem (5.1). Moreover, if $B\left(x^{\prime}, \frac{2}{\lambda}\right) \subset F^{c}$, then $\left(B\left(x^{\prime}, \frac{2}{\lambda}\right) \cup U^{c}\right)^{c}$ is a solution too.

This result is all the more interesting as it gives the following geometrical bounds on some solution of TVL1:

$$
\bigcup_{x: B\left(x, \frac{2}{\lambda}\right) \subset F} B\left(x, \frac{2}{\lambda}\right) \subset U \subset\left(\bigcup_{x: B\left(x, \frac{2}{\lambda}\right) \subset F^{C}} B\left(x, \frac{2}{\lambda}\right)\right)^{C} .
$$

Writing $\mathcal{T}$ for the TVL1 operator, $\mathcal{O}_{2 / \lambda}$ and $\mathcal{F}_{2 / \lambda}$ respectively for the opening and closing operators with radius $\frac{2}{\lambda}$, one can formally write:

$$
\mathcal{O}_{2 / \lambda} \subset \mathcal{T} \subset \mathcal{F}_{2 / \lambda}
$$

This result is somehow optimal since a ball with radius $r<\frac{2}{\lambda}$ vanishes by TVL1. Therefore, it is hopeless to try to get a finer inequality by opening with balls with a smaller radius. Nevertheless, the result is coarse in the sense that it implies a curvature of $\pm \frac{\lambda}{2}$ in regions where the boundary of $U$ is distinct from the boundary of $F$, and not $\pm \lambda$ as predicted by Proposition 5.5. 


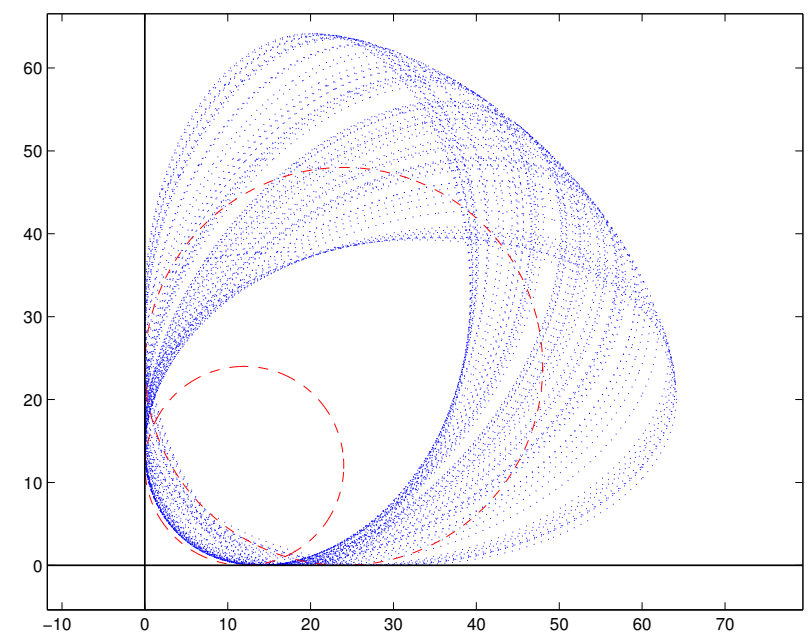

FIG. 5.3. Opening of a corner with discs and calibrable ellipses. Circles of radius $R=1 / \lambda=12$ and $2 / \lambda$ are drawn in red. The ellipses are stretched to the limit of calibrability $(\alpha=1,635 \approx \tilde{\alpha})$ and with maximal curvature $\lambda$, so that they are invariant by TVL1. The arc of circle of radius $1 / \lambda$ (which is the exact solution in this case) is better approximated by the openings with these ellipses than with an opening with a disc of radius $2 / \lambda$.

In fact, we can generalize Proposition 5.17 and give a simpler proof of it by noticing that it is a consequence of the monotonicity Theorem 4.3 (since balls of radius $\frac{2}{\lambda}$ are invariant by TVL1). In the framework of the present paper, one sees that Proposition 5.17 is true when replacing $B\left(x, \frac{2}{\lambda}\right)$ by any set that is invariant by TVL1. As a consequence, one can replace balls in Equation 5.28 by any set that is invariant by TVL1. In order to have better approximations of the TVL1 solutions, we can therefore consider a larger family of structuring elements than just discs.

For instance one may consider ellipses, or any convex calibrable set satisfying the hypothesis of Theorem 5.2, and try to refine the previous inequality. In theory, we are guaranteed that, taking a large enough family, one could describe exactly operators $\mathcal{T}^{+}$or $\mathcal{T}^{-}$: this is indeed a consequence of a famous theorem by Matheron [32] that we now recall.

THEOREM 5.18 (Matheron). Let $\mathcal{P}$ be a translation invariant standard monotone operator. Let us consider $\mathcal{B}=\left\{B \subset \mathbb{R}^{2} / 0 \in \mathcal{P} B\right\}$. Then:

$$
\mathcal{P} X=\left\{x \in \mathbb{R}^{2}, x+B \subset X \text { for some } B \in \mathcal{B}\right\} .
$$

Unfortunately, the family of structuring elements given by this theorem is far too large for a practical use. Indeed any solution of TVL1, up to a translation, is a structuring element! One may thus wonder if a reasonable family could lead to useful approximations of TVL1, yielding curvature values close to $\lambda$. However, the choice of a simple approximating family of structuring elements appears to depend on the considered set. In Figure 5.3, we display the approximation of the evolution of a square corner by openings with calibrable ellipses. Observe also that considering non rotation invariant structuring sets compells us, if we want a rotationally invariant scheme, to add every rotated version of the set in the family: numerically, the computing time would become tremendous. 
Another way to express the result of TVL1 with the help of mathematical morphology is to combine opening-like morphological operators with simple thresholdings. Indeed, an immediate corollary to Theorem 5.14 is the following.

Corollary 5.19. Let $C$ be a bounded, convex set, and $\lambda>0$. Let $C^{1 / \lambda}$ be the opening of $C$ with radius $\frac{1}{\lambda}$. Then:

- If $\frac{\operatorname{Per} C^{1 / \lambda}}{\left|C^{1 / \lambda}\right|}>\lambda$, the solution is $C^{1 / \lambda}$.

- If $\frac{\operatorname{Per} C^{1 / \lambda}}{\left|C^{1 / \lambda}\right|}<\lambda$, the solution is $\emptyset$.

- If $\frac{\operatorname{Per} C^{1 / \lambda}}{\left|C^{1 / \lambda}\right|}=\lambda$, both $C^{1 / \lambda}$ and $\emptyset$ are solutions.

The condition over the ratio perimeter/area appearing in this result essentially means that the energy of the set after the opening is less than the energy of the empty set. In the non convex case we can generalize it by imposing that the energy when keeping the connected component is less than the energy when removing it. Let us mention that a similar thresholding operation has already been proposed in a completely different context, in the framework of morphological connected filters, see [39].

Of course, the approach that would apply openings to non-convex sets is not satisfactory, because it would obviously yield mistakes in locally concave parts. In order to overcome this difficulty, we consider the operators given by an opening followed by a closing or vice versa $\left(\mathcal{F}_{1 / \lambda} \mathcal{O}_{1 / \lambda}\right.$ or $\left.\mathcal{O}_{1 / \lambda} \mathcal{F}_{1 / \lambda}\right)$ with the idea that in regions where the shape is locally convex or concave, Theorem 5.14 applies and these operators would behave correspondingly. Notice also that both operators provide curvature $\pm \lambda$ and also satisfy the same inequality as TVL1, Formula (5.28), namely:

$$
\mathcal{O}_{2 / \lambda} \subset \mathcal{O}_{1 / \lambda} \mathcal{F}_{1 / \lambda} \subset \mathcal{F}_{2 / \lambda} \text { and } \mathcal{O}_{2 / \lambda} \subset \mathcal{F}_{1 / \lambda} \mathcal{O}_{1 / \lambda} \subset \mathcal{F}_{2 / \lambda}
$$

After applying operator $\mathcal{F}_{1 / \lambda} \mathcal{O}_{1 / \lambda}\left(\right.$ or $\mathcal{O}_{1 / \lambda} \mathcal{F}_{1 / \lambda}$ ), we consider every connected component $U_{i}$ of the result, and we test if its energy is lower than the energy of the empty set i.e.:

$$
\text { Per } U_{i}+\lambda\left(\left|U_{i}\right|-2\left|U_{i} \cap F\right|\right) \leq 0 .
$$

Several objections may be raised to this approach. For instance, none of the used morphological operators is self-dual, while TVL1 is. Since there is no ordering between $\mathcal{O}_{1 / \lambda} \mathcal{F}_{1 / \lambda}$ and $\mathcal{F}_{1 / \lambda} \mathcal{O}_{1 / \lambda}$, one cannot even argue that they are good candidates for $\mathcal{T}^{+}$or $\mathcal{T}^{-}$. This objection is often resolved in mathematical morphology by using an alternating sequential filter [40]. This filter consists, given a small value $r_{0}$, in performing alternatively opening and closing of radius $n r_{0}$ until $n r_{0} \geq 1 / \lambda$. When $r_{0} \rightarrow 0$ it is equivalent to start by an opening or a closing, and the algorithm is self-dual.

Another objection is the fact that the thresholding driven by Equation 5.31 might not be monotone in the non convex case. In order to avoid visible artefacts when applying the previous numerical scheme to images, we impose the monotonicity, by removing all connected components of level sets that are included in a removed component.

In Section 6, we detail the proposed scheme and illustrate it by numerical experiments on both synthetic and natural images.

5.4.2. Some remarks about cartoon-texture decomposition. Theorem 5.14 shows that the behavior of the TVL1 model crucially depends on the scale of 
objects. It is interesting to relate this fact with the notion of granulometry used in mathematical morphology in order to study the size of objects:

Definition 5.20. A granulometry is a family of openings $\left\{\gamma_{\mu}\right\}$ depending on a positive parameter $\mu$, that are decreasing functions with respect to $\mu: \mu_{2} \geq \mu_{1}>0 \Rightarrow$ $\gamma_{\mu_{2}} \leq \gamma_{\mu_{1}}$. The cumulative size distribution of a set $F$ is $\mu \mapsto 1-\frac{\left|\gamma_{\mu} F\right|}{|F|}$. Its derivative is called the granulometric spectrum of $F:-\frac{1}{|F|} \frac{d}{d \mu}\left|\gamma_{\mu} F\right|$.

Leaving aside the condition on the ratio perimeter/area in Theorem 5.14, one can notice that the TVL1 model provides a granulometry on the family of convex sets (since $\gamma_{\mu} F=F^{\mu}$ if $\mu \leq \frac{1}{\lambda^{*}}, \emptyset$ otherwise).

In mathematical morphology, granulometric spectrums have notably been used for texture analysis: a texture whose granulometric spectrum is concentrated on small values of $\mu$ is "rough" (that is, composed of tiny objects) whereas if the granulometric spectrum is concentrated on high values of $\mu$ the texture is considered smoother (made with objects at larger scale) (see for instance [40]). The property of granulometries, which is to progressively destroy tiny objects, has proved helpful in practice when trying to discriminate textures. It is reinforced in the TVL1 model by the tresholding on the ratio perimeter/area.

This could explain why the TVL1 model performs surprisingly well in the problem of cartoon-texture decomposition $u+v$. Informally, TVL1 acts on images as a granulometry (which kills small details and small curvatures) followed by a thresholding on the ratio perimeter/area. When the fidelity parameter $\lambda$ varies, objects are altered in function of their granulometry and then vanish when their ratio perimeter/area is too small. The $v$ part contains object with fine granulometry, whereas $u$ contains objects with coarse granulometry (which is what we expect of a cartoon-type image).

It is remarkable that, while the whole community working on cartoon-texture decomposition [33, 41, 36, 13, 14, 15] has investigated norms that tend to favor oscillations in the texture term, one of the best decomposition model [27, 43] is provided by a norm that does not even notice oscillations! The geometric criteria involved by TVL1 have proved relevant in practice, since they have been used in mathematical morphology for years in the study of textures.

6. Numerical experiments. Let us first mention that we do not claim to perform an exact minimization of the TVL1 functional in the discrete case, as is done in [27]. Our purpose here is to illustrate the results of this paper, namely that the TVL1 minimization behaves (exactly in many cases, and at least not very differently in general) like simple morphological operators such as openings or closings. As a result, one obtains a fast and isotropic numerical scheme.

6.1. Naive approach. As described above, the simplest algorithm we propose first consists in performing an opening then a closing $(\mathcal{F O})$, or vice-versa $(\mathcal{O F})$, or an alternate sequential filter (ASF). We then threshold the connected components of the level sets. More precisely:

- Compute the result $M$ of the morphological filter (e.g. the ASF), and set $U=M$.

- For $j=0 . .255$, compute the upper level sets $M^{j}$ and $U^{j}$. For each connected component $C C_{i, j}$ of $M^{j}$, if $C C_{i, j}$ is contained in a connected component of a lower gray level which was thresholded, remove it in $U^{j}$. Else perform the test of Equation (5.31) (remove $C C_{i, j}$ in $U^{j}$ if the result is negative).

- For $j=255 . .0$, compute the lower level sets $M_{j}$ and $U_{j}$. For each connected component $C C_{i, j}$ of $M_{j}$, if $C C_{i, j}$ is contained in a connected component of a 
higher gray level which was thresholded, remove it in $U_{j}$. Else, perform the test of Equation (5.31) (remove $C C_{i, j}$ in $U_{j}$ if the result is negative).

- Return $U$

Observe that the thresholding procedure is self-dual and the only cause of non selfduality could stem from the morphological filter $(\mathcal{O F}, \mathcal{F O}$ or ASF). This procedure could lead to contrast inversion in case of connected components with holes. However, we never observed such a behavior in numerical experiments. Observe that one may show that this phenomenon never happens in the case of concentric circles (annulus) because of the preliminary morphological filter.

6.2. FLST-based approach. Connected components of upper level sets have a tree structure, as well as connected components of lower level sets. Although the previous algorithm can be implemented by imposing monotonicity, it does not fully benefit from this tree structure, and a more elegant approach can be proposed using the Fast Level Set Transform (FLST).

In order to merge the trees of lower and upper level sets, Monasse et al., [34], introduced the concept of shape, and tree of shapes. A shape is defined as the union of a connected component of an upper or lower set together with its holes. The holes of a set $\mathrm{A}$ are defined as the connected components of the complementary set of $\mathrm{A}$ which do not intersect the boundary of the image. It can be shown that these shapes have a tree structure, where the parent-children relationship is given by the inclusion of shapes. This structure results from the fact that two shapes are either disjoint or one is included in the other. To each shape is associated its type indicating whether it stems from a connected component of an upper level set $(\geq \lambda)$ or of a lower level set $(\leq \lambda)$, as well as the value of the associated gray level. An important property of this representation is its self-duality, that is, its invariance with respect to the operation $u \mapsto-u$. This implies in practice that light and dark objects are treated in the same way. A fast algorithm to compute this tree is proposed in [34].

In order to take advantage of the tree of shapes we first notice that the energy of the TVL1 model can be written in a FLST-friendly way. In the discrete case, the energy reads:

$$
\mathcal{E}(u)=\sum_{l=0}^{255}\left(\operatorname{Per} U_{l}+\lambda\left|U_{l} \Delta F_{l}\right|\right)
$$

where $U_{l}$ and $F_{l}$ are the lower level sets of respectively $u$ and $f$. Incidentally, the length of the boundary of $U_{l}$ that coincides with the boundary of the image is not counted in the perimeter Per $U_{l}$ (which amounts to assuming the Neumann conditions $\left.\frac{\partial u}{\partial n}=0\right)$. Now, for a given shape $s$, define $\tilde{s}$ as the set of its proper pixels, that is, pixels which belong to $s$ and not to its children. We obviously have either $\tilde{s} \subset U_{l}$ or $\tilde{s} \subset U_{l}^{C}$. One even has:

$$
U_{l}=\bigcup_{\tilde{s} \subset U_{l}} \tilde{s} \text { and } U_{l}^{C}=\bigcup_{\tilde{s} \subset U_{l}^{C}} \tilde{s}
$$

Given a gray level $l$, the perimeter of a shape $s$ contributes to the perimeter Per $U_{l}$ if and only if $l$ lies in the interval between the value $V_{s}$ of $s$ and the value $V_{p(s)}$ of its parent. Writing co $\left(V_{s}, V_{p(s)}\right)$ for this interval, we therefore have: 


$$
\mathcal{E}(u)=\sum_{l=0}^{255} \sum_{s \text { shape }}\left(\operatorname{Per} s \cdot \mathbf{1}_{l \in \mathrm{co}\left(V_{s}, V_{p(s)}\right)}+\lambda\left|\tilde{s} \cap F_{s l}^{C}\right|\right)
$$

where :

$$
F_{s l}^{C}=\left\{\begin{array}{l}
F_{l}^{C} \text { if } \tilde{s} \subset U_{l} \\
F_{l} \text { if } \tilde{s} \subset U_{l}^{C}
\end{array}\right.
$$

Changing the summation order, we eventually get :

$$
\mathcal{E}(u)=\sum_{s \text { shape }}\left(\sum_{l=0}^{255}\left(\operatorname{Per} s \cdot \mathbf{1}_{l \in \operatorname{co}\left(V_{s}, V_{p(s)}\right)}+\lambda\left|\tilde{s} \cap F_{s l}^{C}\right|\right)\right)
$$

The energy $\mathcal{E}(u)$ is therefore a sum of energies over all the shapes. These energies are not quite independant since the value of a parent has an effect on the energy of its children. As a consequence, one cannot minimize these energies independently. However, one can decrease the global energy by minimizing the energy of the shapes while visiting the tree from the leaves to the root.

More precisely, the thresholding performed at each gray level in the previous algorithm corresponds to modifying the gray value associated with a shape. Given each shape $s$, and assuming its children are fixed (they have already been visited by the algorithm), we move the value $V_{s}$ of the shape towards the value of its parent by one level, if and only if it decreases the energy $(\Delta \mathcal{E}<0)$. The variation of energy is:

$$
\Delta \mathcal{E}=- \text { Per } s+\lambda\left|\tilde{s} \cap F_{s, V_{s} \pm 1}^{C}\right|+\sum_{\begin{array}{c}
c \text { child of } \mathrm{s} \\
\text { with same type }
\end{array}} \text { Per } c-\sum_{\begin{array}{c}
c \text { child of s } \\
\text { with opposite type }
\end{array}} \text { Per } c
$$

where $V_{s} \pm 1$ is the new gray value to be given to $s$. Recall that if a shape $s$ is of inferior type, then the value of its parent is above the value of $s$, and if $s$ is of superior type, the value of its parent is below. Therefore the gray value of $s$ is getting closer to the values of the children with opposite type, but is getting further from the values the children with same type.

Notice that this procedure is very similar to the one proposed by Dibos et al. in [28] in order to simulate the TV flow by using the FLST, and later by Darbon in [26] for TVL1. However the difference is that we start with the output of an ASF filter and compare it to the original image. A first consequence is that the criterion to decide whether or not the gray level associated with a shape should be modified is different. A second difference is that our approach is legitimated by our theoritical study. In particular, whereas Dibos and Darbon do not impose any condition on the curvature of the output, we know that the shapes provided by the ASF filter are somehow "good candidates" to be solutions of TVL1.

This procedure decreases the energy and in most case provides the same results as the simple algorithm presented in Section 6.1 and dealing directly with level sets. The algorithm relying on the FLST is very fast compared to the simple algorithm and even competitive with Darbon-Sigelle's algorithm [27] : using square as structuring element it took $1.27 \mathrm{~s}$ to filter a $512 \times 512$ image with $\lambda=1 / 3$, using an Intel Core2 Duo E8400, 
$4 \mathrm{~Gb}$ of RAM. Notice that in this experiment, half the computation time is due to the openings and closings (ASF filter). As a comparison, Darbon-Sigelle's algorithm takes $0.96 \mathrm{~s}$ using 4-connectivity. Using isotropic schemes (i.e. balls as structuring elements versus 8-connectivity), the trend is inverted since Darbon-Sigelle's algorithm takes longer using a more complicated connectivity $(1.71 s)$ whereas the FLST -based scheme is not affected at all $(1.27 \mathrm{~s})$. However, let us recall again that we do not perform exact minimization.

6.3. Commentary. In this section we discuss the results of the FLST-based algorithm. It should be noted that in order to compare this algorithm with existing TVL1 algorithms, we should focus on schemes that satisfy the coarea formula. For instance, when using a gradient descent formulation, numerical instabilities compells us to regularize the total variation by replacing $\sqrt{u_{x}^{2}+u_{y}^{2}}$ with $\sqrt{\epsilon^{2}+u_{x}^{2}+u_{y}^{2}}$ in the divergence term. Although $\epsilon$ is assumed to be very small, this implies that the scheme does not satisfy the coarea formula. As a matter of fact, in the discrete case, even when $\epsilon=0$, the coarea formula is not satisfied. As a consequence, new grey levels appear and characteristic functions of sets are blurred. Therefore, it is not possible to experimentaly check e.g. curvature values because of uncertainty on the boundary of the smoothed sets (see Figure 6.1).

We therefore compare our results with Darbon-Sigelle's algorithm [27] since, to our knowledge, it is the only algorithm that satisfies the coarea formula. Observe however that this algorithm is anisotropic. Its authors propose two different schemes: one relying on 4-connectivity and the other one relying on 8-connectivity, the latter being more isotropic. In case of 4-connectivity, the scheme is actually consistent with an anisotropic total variation formally defined as: $\int\left(\left|u_{x}\right|+\left|u_{y}\right|\right)$ instead of $\int \sqrt{u_{x}^{2}+u_{y}^{2}}$. This kind of anisotropic, or crystalline, total variation is the framework of intensive research (among which [19],[23], [17]). Let us mention that one can define an anisotropic perimeter as well as an anisotropic curvature and that similar results as those of Lemma 5.11 can be stated [19]. In our case the associated Wulff shape is a square. It is not the purpose of this paper to explore the links between anisotropic total variation and the results of Section 4, but we believe (and numerical experiments tend to confirm it) that our results still hold with anisotropic total variation when replacing opening with balls by opening with squares. Therefore, the reader should keep in mind, while looking at the result with Darbon-Sigelle's algorithm connectivity 4 , that the opening should be performed with squares instead of balls.

Figure 6.1 shows the evolution of simple convex and non-convex shapes. First, notice that results of rows 2 and 3 (respectively from Darbon-Sigelle's algorithm and our approach when using square structuring elements) are remarkably similar (although a few pixels may differ). Observe also that, as it is well known, the finite difference implementation of the fourth row exhibits blur. Last, we can notice that results displayed on the last row, using balls as structuring elements, are the only ones being both isotropic and with sharp edges.

Figure 6.2 and 6.3 illustrate the ability of the algorithm to provide isotropic results when using balls as structuring elements and provides comparisons with DarbonSigelle's algorithm using 8-connectivity on natural images. Some details may differ but on the whole the results look very similar. Incidentally, let us notice that using 8-connectivity with Darbon-Sigelle's algorithm yields, as it is well-known, slightly anisotropic results, but this anisotropy becomes visible only at very low values of $\lambda$ (which are unrealistic when denoising images). The isotropy of the FLST based 
algorithm, is a direct consequence of the isotropy of the scheme used to perform the openings. Here, the scheme is the one included in Megawave and yields very satisfying results. However, more sophisticated implementations could be considered.

Figure 6.4 displays another experiment on a natural image. In practice, the alternating sequential filter is not exactly self-dual since a finite step $r_{0}=1$ has to be chosen. As a consequence, the result in some specific areas may depend on the order of the ASF. Figure 6.5 shows the "granulometry property" of the algorithm.

7. Adaptive TVL1. In the remainder of the paper, we consider the following spatially varying version of the TVL1 problem:

$$
\inf _{u} \int_{\mathbb{R}^{2}}|D u|+\int_{\mathbb{R}^{2}}|u-f| \lambda(x) d x
$$

in which the fidelity parameter $\lambda$ can be locally adapted to the content of the image. Typically, in textured areas, one may want to use a higher fidelity parameter in order to preserve fine details, while smoothing homogeneous regions. Another application may be the denoising of medical images where one knows in advance where objects of interest are and one wants to preserve them.

The aim of this section is to show that there are (locally) two options when using adaptive TVL1 and defining $\lambda(x)$ : either one bounds $\lambda(x)$ and gets regular solutions $(\lambda(x)$ giving a bound on the curvature), or one lets $\lambda$ unbounded and then any shape can be preserved by TVL1, including those with corners.

It will sometimes be more convenient to consider the weight $\lambda$ as a density with respect to the Lebesgue measure: $\lambda(x) d x=d \mu$. For technical reasons we will assume that $\lambda \in L_{l o c}^{1}$ and that

$$
\exists c>0, \text { a.e. } x \in \mathbb{R}^{2}, \lambda(x) \geq c>0 .
$$

Notice that under these assumptions, the space of $\mu$-integrable functions, that we denote by $L_{\lambda}^{p}$, enjoys the usual properties of Lebesgue spaces (duality $L_{\lambda}^{p}-L_{\lambda}^{p^{\prime}}$, density of smooth functions, etc.).

When $\lambda$ is constant, Problem (7.1) amounts to the regular TVL1 model, in which case we shall say that the problem is stationary .

Proposition 7.1 (Existence). Assume condition (7.2) is true. Then there exists (at least) one solution to Problem (7.1). Let us define:

$$
T: L_{\lambda}^{1} \rightarrow \mathcal{P}\left(L_{\lambda}^{1}\right)
$$

the operator which maps $f$ to the set of solutions $T f=\left\{u \in L_{\lambda}^{1}\right.$, $u$ is a solution of (4.1) $\}$. Then the set $T f$ is convex and closed in $L_{\lambda}^{1}$.

Proof. The proof relies on the direct method of the calculus of variations. Let $u_{n}$ be a minimizing sequence. Then,

$$
\min (c, 1)\left(\left|u_{n}\right|_{T V}+\int_{\mathbb{R}^{2}}\left|f-u_{n}\right|\right) \leq\left|u_{n}\right|_{T V}+\int_{\mathbb{R}^{2}}\left|f-u_{n}\right| d \mu \leq C
$$

thus we can extract a subsequence (still denoted as $u_{n}$ ) which converges to $u \in L^{1}$ in $L^{1}$ and $D u_{n} \rightarrow D u$ in the sense of measure convergence. Up to another extraction, we can even assume that the sequence $u_{n}(x)$ converges to $u(x)$ for almost every $x \in \mathbb{R}^{2}$. But then by semicontinuity and Fatou's lemma: 

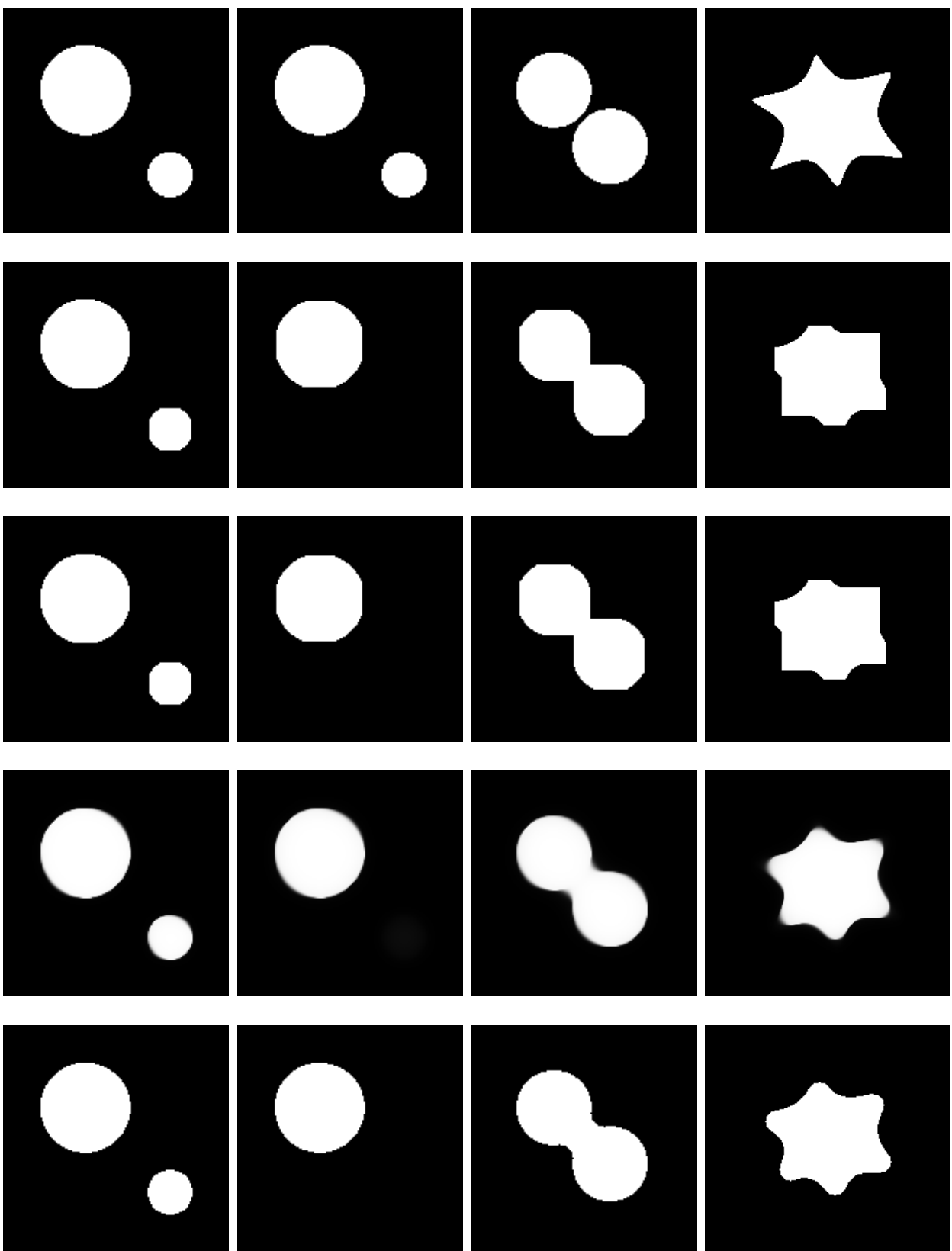

Fig. 6.1. From top to bottom. First row: original image. Second row: result obtained with Darbon-Sigelle's algorithm (connectivity 4). Third row: result of the FLST based algorithm, i.e. an alternating sequential filter (alternated opening/closing with increasing radius, the structuring element being a square of side $2 / \lambda$ ) completed with a thresholding on the shapes. Fourth row: result of the gradient descent scheme (300 000 iterations, $d t=0.1)$. Fifth row: result of the FLST-based algorithm with euclidean balls as structuring elements. All pictures were computed with $\lambda=1 / 9$, except the first column ( $\lambda=1 / 6$, the small component is kept, contrary to the second column). Notice the blurry effect introduced by the the gradient descent. 


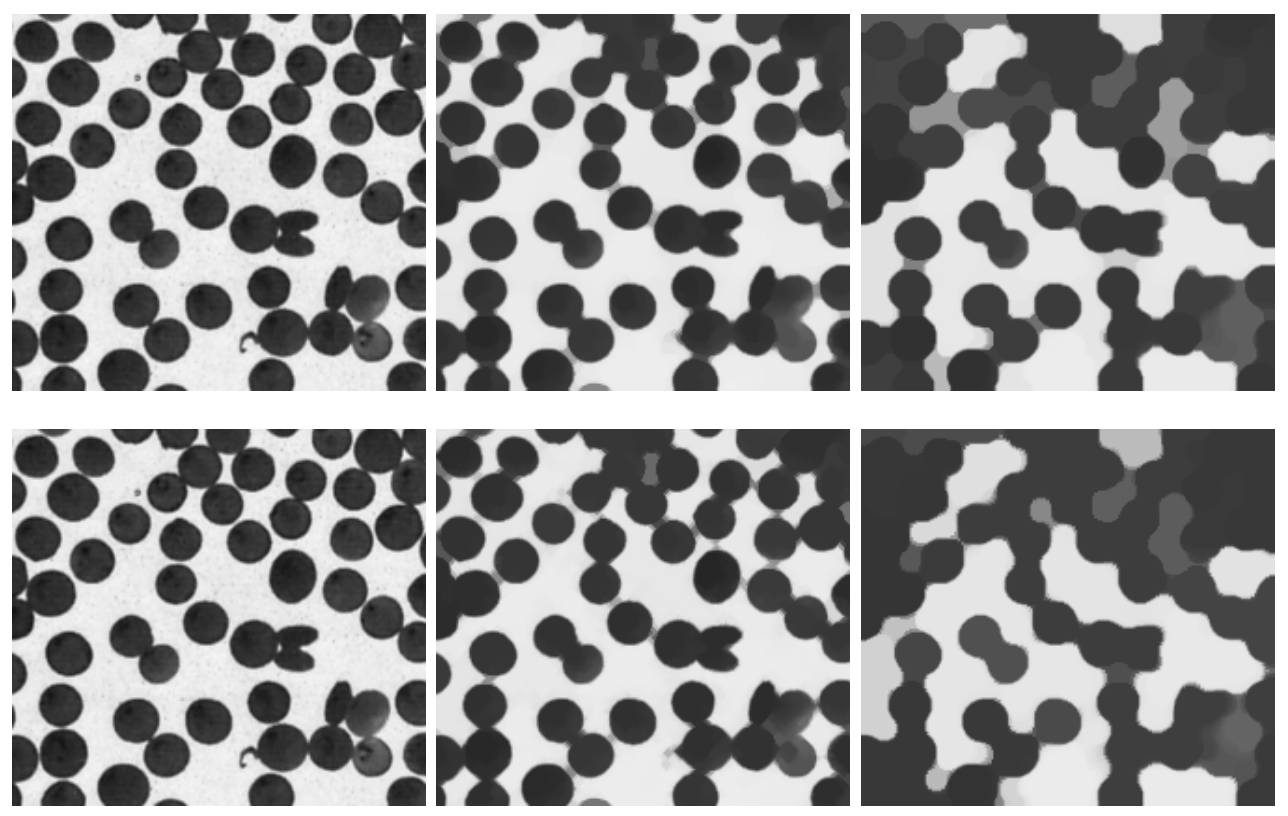

FIG. 6.2. Isotropy. The upper row illustrates the behavior the Darbon-Sigelle algorithm using 8-connectivity on a detail of a Brodatz texture (from left to right: original image, result of the algorithm with increasing values of $\lambda$ ) Lower row: behavior of the FLST-based filter using euclidean balls followed by a thresholding. Notice that the anisotropy of Darbon-Sigelle's scheme is not very visible, even at low values of $\lambda$.
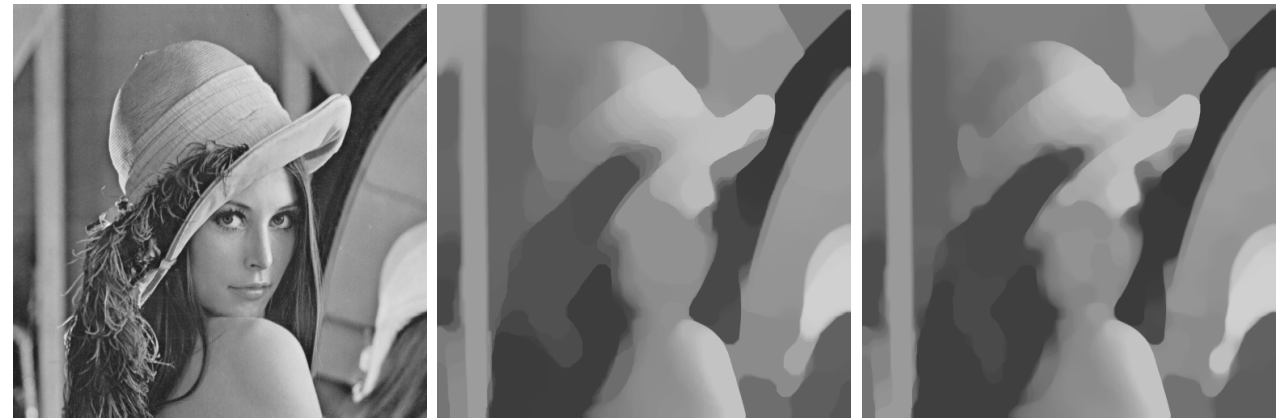

FIG. 6.3. Isotropy again. Left: original image. Middle: result of Darbon Sigelle's algorithm using 8-connectivity $(\beta=4.6)$. Using 8-connectivity provides more isotropic results than using 4-connectivity. However, especially for very low values of $\lambda$, it is not as isotropic as the scheme proposed in this paper when using balls as structuring element, as shown on the right image $(\lambda=$ $1 / 11$ ).

$$
\liminf _{n}\left(\left|u_{n}\right|_{T V}+\int_{\mathbb{R}^{2}}\left|f-u_{n}\right| d \mu\right) \geq|u|_{T V}+\int_{\mathbb{R}^{2}}|f-u| d \mu
$$

and $u$ is a solution of Problem 7.1.

Proposition 7.2. The energy reformulates: 

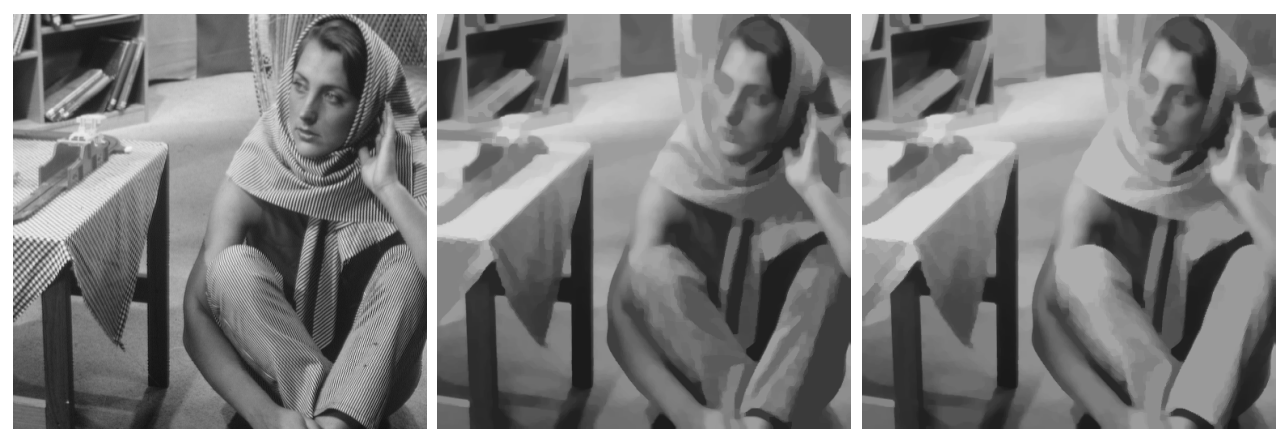

FIG. 6.4. Chessboard dilemma. From left to right: original image, results of the FLST based algorithm $(\lambda=1 / 3$ in the first case the alternating sequential filter starts with an opening, and in the second one it starts with a closing). The ASF is not exactly self-dual and in some specific cases the result visibly depends on the chosen order. However, this does not necessarily contradict the TVL1 model since in case of the chessboard dilemma, the solution of the model is not unique.
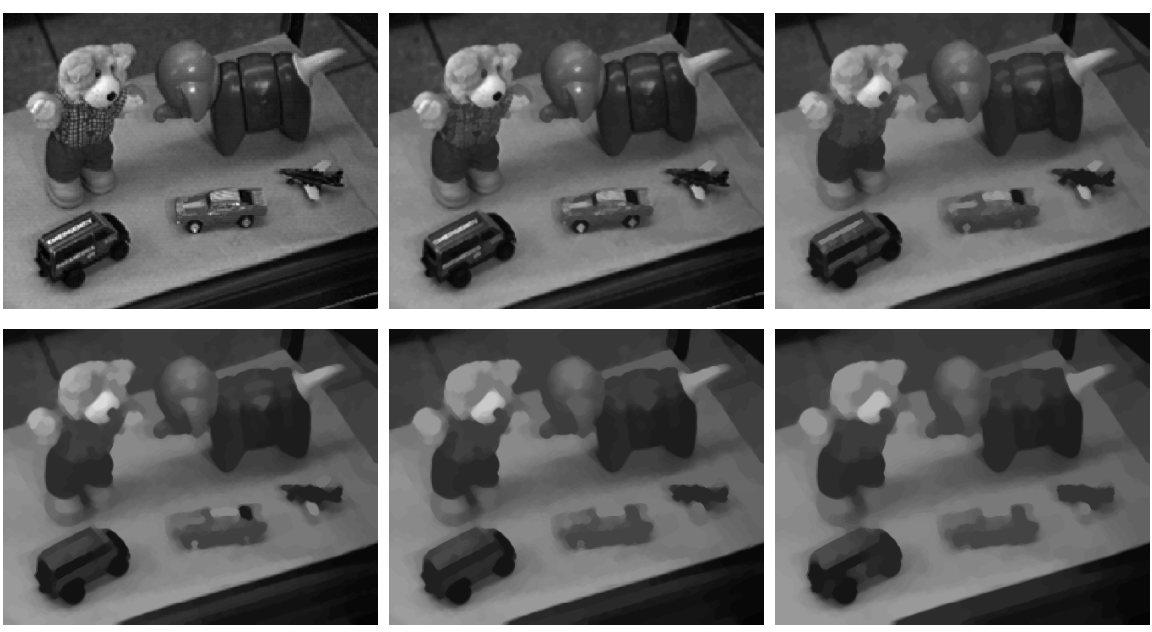

FIG. 6.5. Scale space using the FLST-based algorithm. From left to right, top to bottom: original image, result of the FLST-based algorithm $(\lambda=1,1 / 2,1 / 3,1 / 4,1 / 5)$. Observe how details vanish one after the other, in function of their size: the shirt, the eyes of the teddy-bear, one wing of the plane... This illustrates the "improved granulometry" property of the TVL1 model: objects evolve according to an opening/closing and then vanish depending on a geometric criterion.

$$
\mathcal{E}(u)=\int_{-\infty}^{+\infty} \operatorname{Per}\{x, u(x)>t\}+\mu(\{x, u(x)>t\} \triangle \mid\{x, f(x)>t\}) d t .
$$

We are thus led to study the geometric problem:

$$
\inf _{U \subset \mathbb{R}^{2}} \mathcal{E}_{G}(U):=\operatorname{Per} U+\mu(U \triangle F)
$$

where $\mu \in \mathbb{R}$ and $F_{\mu}=\{x, f(x)>\mu\}$. Notice that when willing to insist on the dependance on the data $F$, we shall write $\mathcal{E}_{G}(U, F)$.

Proof. 


$$
\begin{aligned}
\int_{\mathbb{R}^{2}}|u-f| d \mu & =\int_{\mathbb{R}^{2}}\left(\int_{f(x)}^{u(x)} d t\right) \chi_{f(x)<u(x)}+\left(\int_{u(x)}^{f(x)} d t\right) \chi_{u(x)<f(x)} d \mu \\
& =\int_{\mathbb{R}^{2}} \int_{-\infty}^{+\infty}\left(\chi_{f(x) \leq t<u(x)}+\chi_{u(x) \leq t<f(x)}\right) d t d \mu
\end{aligned}
$$

But

$$
\{x,(u(x)>t \geq f(x)) \text { or }(u(x) \leq t<f(x))\}=\{x, u(x)>t\} \triangle\{x, f(x)>t\}
$$

By Fubini's theorem:

$$
\begin{aligned}
\int_{\mathbb{R}^{2}}|f-u| d \mu & =\int_{-\infty}^{+\infty} \int_{\mathbb{R}^{2}} \chi_{\{x, u(x)>t\} \triangle\{x, f(x)>t\}} d \mu d t \\
& =\int_{-\infty}^{+\infty} \int_{\mathbb{R}^{2}} \mu(\{x, u(x)>t\} \triangle\{x, f(x)>t\}) d t
\end{aligned}
$$

7.1. Monotonicity. In this section we generalize the monotonicity result (Theorem 4.3), following the steps of [44] for the stationary case but giving different proofs.

Lemma 7.3. Let $F \subset G \subset \mathbb{R}^{2}$ with finite $\mu$-measure, let $U_{1}, U_{2}$ be corresponding solutions, and $U_{\wedge}=U_{1} \cap U_{2}$. We have:

$$
\mathcal{E}_{G}\left(U_{1}, F\right)-\mathcal{E}_{G}\left(U_{\wedge}, F\right) \geq \mathcal{E}_{G}\left(U_{1}, G\right)-\mathcal{E}_{G}\left(U_{\wedge}, G\right)
$$

where $\mathcal{E}_{G}(U, F)$ is the geometric energy of $U$ (see Equation (7.7)) with data $F$.

Proof. Since the perimeter parts of the energies are equal, it is sufficient to prove the following inequality for the fidelity terms:

$$
\mu\left(U_{1} \triangle F\right)-\mu\left(\left(U_{1} \cap U_{2}\right) \triangle F\right) \geq \mu\left(U_{1} \triangle G\right)-\mu\left(\left(U_{1} \cap U_{2}\right) \triangle G\right)
$$

Since $G, F$ and $U_{1}$ have finite measure we can write:

$$
\begin{aligned}
\mu\left(U_{1} \Delta G\right) & =\mu\left(U_{1} \cup G\right)-\mu\left(U_{1} \cap G\right) \\
& =\mu\left(U_{1} \cup F\right)+\mu\left(\left(G \backslash\left(F \cup U_{1}\right)\right)-\left(\mu\left(U_{1} \cap F\right)+\mu\left(U_{1} \cap(G \backslash F)\right)\right.\right. \\
& =\mu\left(U_{1} \Delta F\right)+\mu\left((G \backslash F) \cap U_{1}^{C}\right)-\mu\left(U_{1} \cap(G \backslash F)\right)
\end{aligned}
$$

Therefore:

$$
\mu\left(U_{1} \Delta F\right)-\mu\left(U_{1} \Delta G\right)=\mu\left(U_{1} \cap(G \backslash F)\right)-\mu\left((G \backslash F) \cap U_{1}^{C}\right)
$$

and similarly:

$$
\mu\left(\left(U_{1} \cap U_{2}\right) \Delta F\right)-\mu\left(\left(U_{1} \cap U_{2}\right) \Delta G\right)=\mu\left(U_{1} \cap U_{2} \cap(G \backslash F)\right)-\mu\left((G \backslash F) \cap\left(U_{1} \cap U_{2}\right)^{C}\right)
$$

Notice that the right side of the last two equations is a non-decreasing function of $U_{1}$ (resp. $U_{1} \cap U_{2}$ ), hence the result. 
Lemma 7.4. The mapping $U \mapsto \mu(U \triangle F)$ is submodular, ie:

$$
\mu(U \cap V \triangle F)+\mu(U \cup V \triangle F) \leq \mu(U \triangle F)+\mu(V \triangle F) .
$$

Proof. Let us write: $u=\chi_{U}, v=\chi_{V}$ and notice that $u^{2}=u, v^{2}=v$.

We have:

$$
\begin{gathered}
|u v-f|=|(u-f) v+(1-v)(v-f)| \leq v|u-f|+(1-v)|v-f|, \\
|u+v-u v-f|=\left|(u-f)(1-v)-v f+v^{2}\right| \leq(1-v)|u-f|+v|v-f| .
\end{gathered}
$$

Therefore: $|u v-f|+|u+v-u v-f| \leq|u-f|+|v-f|$. Integration with respect to the measure $\mu$ gives the result.

Theorem 7.5 (Pseudo-monotonicity). Let $S_{1} \subset S_{2} \subset \mathbb{R}^{2}$, and $U_{i}, i=1,2$ be a solution of the adaptive geometric problem associated to $S_{i}$. Then

- $U_{\wedge}=U_{1} \cap U_{2}$ is a solution associated to $S_{1}$

- $U_{\vee}=U_{1} \cup U_{2}$ is a solution associated to $S_{2}$.

Proof. We proceed as in [44].

$$
\begin{aligned}
0 & \geq \mathcal{E}_{G}\left(U_{1}, S_{1}\right)-\mathcal{E}_{G}\left(U_{\wedge}, S_{1}\right) \text { by optimality of } U_{1} \\
& \geq \mathcal{E}_{G}\left(U_{1}, S_{2}\right)-\mathcal{E}_{G}\left(U_{\wedge}, S_{2}\right) \text { by Lemma } 7.3 \\
& \geq \mathcal{E}_{G}\left(U_{\vee}, S_{2}\right)-\mathcal{E}_{G}\left(U_{2}, S_{2}\right) \\
& \geq 0 \text { by optimality of } \mathcal{E}_{G}\left(U_{2}, S_{2}\right) .
\end{aligned}
$$

The third inequality is a consequence of the submodularity of the perimeter and the fidelity term. As a result, all inequalites are in fact equalities and $U_{\wedge}\left(\right.$ resp. $\left.U_{\vee}\right)$ is a minimizer associated to $S_{1}$ (resp. $S_{2}$ ).

A consequence of this monotonicity property is the geometric equivalence theorem.

THEOREM 7.6 (Geometric equivalence). Let $f \in L_{\lambda}^{1}$. The following assertions are equivalent:

(i) $u$ is solution of (7.1)

(ii) Almost every level set $U_{\rho}$ of $u$ is solution of (7.7).

Proposition 7.7 (Contrast invariance). Let $g$ be an increasing $C^{1}$-diffeomorphism with $\sup \left|g^{\prime}\right|<+\infty$, and $f \in L_{\lambda}^{1}$. Then:

$$
T_{\lambda}(g \circ f)=g\left(T_{\lambda} f\right) .
$$

7.2. Smoothness of the boundary. The following results explain that, as in the stationary case, when $\lambda$ is bounded, the boundary of the solutions is smooth.

Proposition 7.8. Assume $\lambda$ is essentialy bounded. Let $F \subset \mathbb{R}^{2}$, and $U \subset \mathbb{R}^{2}$ be a solution of the TVL1 problem associated to $F$. Then $\partial U$ coincides with the reduced boundary of $U$ in the sense that $\mathcal{H}^{1}\left(\partial U \backslash \partial^{*} U\right)=0$, and the latter is a $C^{1,1}$ hypersurface.

Proof. It is the same as the stationary case. We let $M=\|\lambda\|_{\infty}$ and notice that $U$ is a strong $M$-minimizer [9]. 
Notice that, if we assume $\lambda$ to be only locally bounded, the result holds locally.

In the following proposition, we assume that the weight has bounded variation, which ensures that it has a well-defined trace on the boundary of the solution. This trace controls the curvature of the solution.

THEOREM 7.9. Let us assume that $\lambda \in B V_{l o c} \cap L_{l o c}^{\infty}$. Let $U \subset \mathbb{R}^{2}$ be a bounded open set of class $C^{1,1}$, solution of the TVL1 problem for some $F \subset \mathbb{R}^{2}$. Then:

$$
\left|\kappa_{\partial U}(x)\right| \leq \lambda(x) \mathcal{H}^{1} \text { a.e. } x \in \partial U .
$$

Proof. Let $V=D \times I \subset \mathbb{R}^{2}$ be an open set intersecting $\partial U$, such that $U \cap V$ is the hypograph of a $C^{1}$ function, and $h \in C_{0}^{1}(V)$. Let $\alpha>0$ small, and $\psi_{\alpha}(x):=$ $x+\alpha h(x) \nu(x)$, where $\nu \in C^{1}\left(V, \mathbb{R}^{2}\right)$ is a vector field such that $|\nu|=1$ on $U$, and coincides with the outer normal of $U$ on $\partial U \cap V$. Let us extend $\psi_{\alpha}$ by $\psi_{\alpha}(x)=x$ for $x \notin U$. Notice that when $\alpha$ is small enough, $\psi_{\alpha}$ is a $C^{1}$ diffeomorphism of $\mathbb{R}^{2}$ in $\mathbb{R}^{2}$, such that $\psi_{\alpha}(U \cap V) \subset U \cap V$. Let us write $U_{\alpha}:=\psi_{\alpha}(U)$. Then, if $h$ is non-positive, since $U$ and $U_{\alpha}$ coincide outside $V$ and $U \cap V \subset U_{\alpha} \cap V$ :

$$
0 \leq \frac{\mathcal{E}_{G}\left(U_{\alpha}\right)-\mathcal{E}_{G}(U)}{\alpha}=\frac{\operatorname{Per} U_{\alpha}-\operatorname{Per} U}{\alpha}+\frac{\mu\left(U_{\alpha} \backslash U\right)}{\alpha}
$$

(Note that $\mathcal{E}_{G}$ refers here to the geometric energy associtated with data $U$.)

The first term tends to $\int_{\partial U} \kappa_{\partial U} h d \mathcal{H}^{1}$ for $\alpha \rightarrow 0$, so let us focus on the second one. Since:

$$
\int_{U_{\alpha}} \lambda(y) d y=\int_{U} \lambda(x+\alpha h(x) \nu(x)) \operatorname{det}(I+\alpha D(h \nu)) d x
$$

we have:

$\frac{\mu\left(U_{\alpha}\right)-\mu(U)}{\alpha}=\int_{U} \frac{\lambda(x+\alpha \nu h)-\lambda(x)}{\alpha} \operatorname{det}(I+\alpha D w) d x+\int_{U} \lambda(x)\left(\frac{\operatorname{det}(I+\alpha D(h \nu))-1}{\alpha}\right) d x$

The limit of this expression for $\alpha \rightarrow 0^{+}$(see Lemmas 7.13 and 7.14) is:

$$
\int_{U}(D \lambda, h \nu)+\int_{U} \lambda \operatorname{div}(h \nu)=-\int_{\partial U} \lambda h \nu d \mathcal{H}^{1}
$$

Eventually, for all $h \leq 0$ with support in $V$ :

$$
0 \leq \int_{\partial U}\left(\kappa_{\partial U}-\lambda\right) h \nu d \mathcal{H}^{1}
$$

thus $\kappa(x) \leq \lambda(x) \mathcal{H}^{1}$ a.e. $x \in V \cap \partial U$.

In a similar way, taking $h \geq 0$, we have $U_{\alpha} \subset U$ and $\kappa(x) \geq-\lambda(x) \mathcal{H}^{1}$ a.e. $x \in$ $V \cap \partial U \square$

As in the stationary case, on can prove that in neighborhoods where the boundaries of $F$ and $U$ do not coincide, the curvature of $U$ is actually equal to $\pm \lambda(x), \mathcal{H}^{1}$ a.e.x (whether the point $x$ lies inside or outside $F$ ).

REMARK 7.10. It is easy to see (consider for instance a disc), that even in the convex case, the condition $\left(\frac{\operatorname{Per} C}{\mu(C)} \leq 1, \kappa_{\partial C}(x) \leq \lambda(x) \mathcal{H}^{1}\right.$ a.e. $\left.x \in \partial C\right)$ of Theorem 5.9 is necessary but not sufficient for a general weight $\lambda$. 
However, it is possible to compare to the stationary case to get sufficient conditions. If:

$$
\kappa_{\partial C}(x) \leq \operatorname{ess} \inf _{y \in C} \lambda(y) \text { and } \frac{\operatorname{Per} C}{|C|} \leq \operatorname{ess} \inf _{y \in C} \lambda(y),
$$

then $C$ is solution. (write: Per $U+\int_{\emptyset} d \mu=\operatorname{Per} U+c \int_{\emptyset} d x \leq \operatorname{Per} V+c \int_{C \backslash V} d x \leq$ Per $\left.V+\int_{C \backslash V} d \lambda\right)$

7.3. Corners with adaptive TVL1. The last subsection showed that in regions where $\lambda$ is bounded, the boundary of the solution is regular. In the stationary case it is well-known that one cannot preserve sharp corners while minimizing the total variation in the ROF model (see for instance [33]) or in the TVL1 model (Proposition 5.4). On the contrary, one might want, in certain regions of an image, to preserve corners or tiny details.

In [16], it was proved that any bounded set $F \subset \mathbb{R}^{2}$ with finite perimeter has a variational curvature, i.e. there exists a non-negative weight $\lambda(x)$ such that $F$ is solution of the problem:

$$
\inf _{U \subset F} \operatorname{Per} U-\int_{U} \lambda(x) d x
$$

In view of the geometrical analysis of this paper, this clearly implies that when the data $F$ is convex (for instance a square), there exists a weight $\lambda(x)$ such that TVL1 preserves this data. The idea in [16] is to consider an increasing sequence $\lambda_{n} \rightarrow+\infty$ and consider the associated solutions of the stationary problem (which gives a nondecreasing sequence of sets $E_{i}$ ). Then define $\lambda(x)=\lambda_{i}$ if $x \in E_{i} \backslash E_{i-1}$ (see Figure 7.1). We see that no condition is imposed on the weight $\lambda$ outside of the convex set since $E_{i} \subset F$.

It seems difficult to generalize this result to non convex shapes. However, in view of image processing applications, we can extend it to polygons.

Proposition 7.11. Let $F \subset \mathbb{R}^{2}$ be a simple polygon (not necessarily convex). Then there exists a weight $\lambda \in L^{1}$ such that $F$ is invariant by adaptive TVL1. Moreover, given a distance $r>0$, one can assume that $\lambda(x)=0$ for $\operatorname{dist}(x, F)>r$.

Let us remind the reader that a simple polygon is a polygon whose boundary does not cross itself. The last part of the proposition means that the choice of $\lambda$ is almost local, and that one can combine weights associated with different objects provided they are far enough from one another.

Proof. Let $X=\left\{\left(x_{1}, y_{1}\right), \ldots,\left(x_{N}, y_{N}\right)\right\}$ denote the set of vertices of the polygon $F$. Set $0<\alpha<\min \left(\left\{\left|x_{i}-x_{j}\right|, / x_{i} \neq x_{j} \mid\right\} \cup\left\{\left|y_{i}-y_{j}\right|, / y_{i} \neq y_{j} \mid\right\}\right)$ and define a grid $\alpha \mathbb{Z}^{2}$. Define its open cells by:

$$
\forall(i, j) \in \mathbb{Z}^{2}, V_{i, j}=\left\{(x, y) \in \mathbb{R}^{2}, \alpha i<x<\alpha(i+1), \alpha j<y<\alpha(j+1)\right\} .
$$

Since the number of segments is finite, we can assume, up to a rotation of the axes, that no segment is parallel with the axes (therefore the edges of $F$ intersect with the grid only at isolated points). Moreover, by choice of $\alpha$ the adherence of each $V_{i, j}$ contains at most one vertex, and either $F \cap V_{i, j}$ is convex or $F^{C} \cap V_{i, j}$ is convex (since it is the intersection of the triangle defined by the vertex and its two neighbors with the convex cell $V_{i, j}$ ), see Figure 7.1.

In each cell $V_{i, j}$ such that $F \cap V_{i, j} \neq \emptyset$, consider the restricted TVL1 problem (we assume for clarity that $F \cap V_{i, j}$ is convex, but by self-duality of the model it deals 
with the other case as well):

$$
\inf _{U \subset F \cap V_{i, j}} \operatorname{Per} U-\int_{U} \lambda_{i, j}(x) d x
$$

By the result of [16], one can find a weight $\lambda_{i, j}$ such that $F \cap V_{i, j}$ is a solution, and $\lambda_{i, j}$ vanishes outside $F \cap V_{i, j}$.

Now we collect the weights and define a function $\lambda$ such that $\lambda \equiv \lambda_{i, j}$ on $V_{i, j}$ for all $(i, j) \in \mathbf{Z}^{2}$. Then, since by assumption the edges of $F$ intersect the grid at most at isolated points:

$$
\left|D \chi_{F}\right|\left(\mathbb{R}^{2}\right)=\sum_{(i, j) \in \mathbb{Z}^{2}}\left|D \chi_{F}\right|\left(V_{i, j}\right)
$$

By summing the inequalites on cells, for any $U \subset \mathbb{R}^{2}$ :

$$
\left|D \chi_{F}\right|\left(\mathbb{R}^{2}\right)=\sum_{(i, j) \in \mathbb{Z}^{2}}\left(\left|D \chi_{U}\right|\left(V_{i, j}\right)+\int_{\left(F \cap V_{i, j}\right) \Delta\left(U \cap V_{i, j}\right)} \lambda(x) d x\right) \leq\left|D \chi_{U}\right|\left(\mathbb{R}^{2}\right)+\int_{(F \Delta U)} \lambda(x) d x
$$

Therefore $F$ is a solution with such $\lambda$.

To be coherent with our framework (Equation 7.2), once such $\lambda$ is found, one can even replace $\lambda$ by $\lambda+c$.
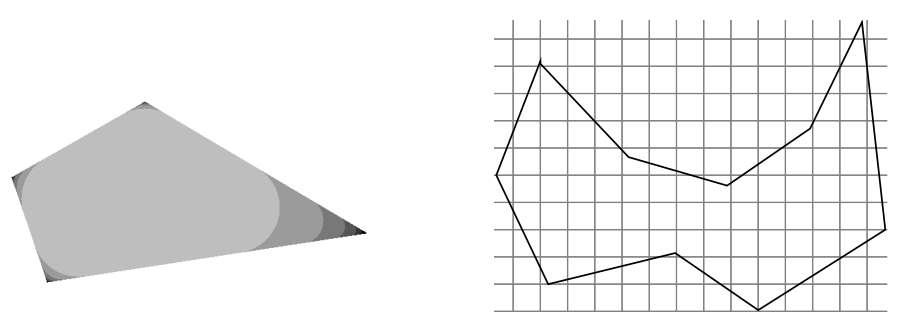

FIG. 7.1. (Left) A convex polygon and its solution $E_{i}$ with TVL1 using an increasing sequence $\lambda_{i}$ (the darker the greater value of $\lambda$ ). In [16], it is proved that using precisely the weights $\lambda_{i}$ in the corresponding sets leads to a perfect preservation of the convex set. (Right) Generalization to non convex polygons. If the boundary is simple, one can define a grid such that for each cell $V_{i, j}$, $F \cap V_{i, j}$ is convex or $F^{C} \cap V_{i, j}$ is convex.

7.4. Generalized Cheeger sets and $\lambda$-calibrable sets. Interestingly enough, the adaptive TVL1 model is related to the notion of generalized Cheeger set studied in [21], [20], and [22].

Given a nonempty open bounded set $F \subset \mathbb{R}^{2}$, determine:

$$
h_{F}:=\inf _{X \subset F} \frac{\int_{F}\left|D \chi_{X}\right|(x)}{\int_{X} \lambda(x) d x}=\frac{\operatorname{Per} X}{\mu(X)}
$$

Any minimizer $X$ of this problem is called a generalized Cheeger set of $F$. If $F$ itself is a minimizer, we say that $F$ is Cheeger in itself.

Notice that $F$ is Cheeger in itself if and only if it is solution of the problem:

$$
\min _{X \subset F} \operatorname{Per} X-\lambda_{F} \mu(X)
$$


with $\lambda_{F}=\frac{\text { Per } F}{\mu(F)}$.

We can also extend the notion of calibrable sets to this framework.

DeFinition 7.12. Let $E$ be a bounded set with finite perimeter. We say that $E$ is $\mu$-calibrable if there exists a vector field $\xi \in X_{\infty}$ with $\|\xi\|_{\infty} \leq 1$ such that - div $\xi=\lambda_{E} \chi_{E} \lambda(x)$ in $\mathcal{D}^{\prime}\left(\mathbb{R}^{2}\right)$ for some $\lambda_{E}$, and $\left(\xi, D \chi_{E}\right)=\left|D \chi_{E}\right|$ as measures in $\mathbb{R}^{2}$.

With this definition, Propositions 3.2 and 3.3 are true when replacing the Lebesgue measure with $\mu$ (see Appendix B).

As a consequence, a $\mu$-calibrable set $F$ is $(\mu$-) Cheeger in itself, and the converse is true when $F$ is convex.

Replacing $\mu$ by $\alpha \mu(\alpha>0)$, one can look for the sudden disparition of $F$ when $\alpha$ varies. As in the stationary case, one can show that every $\mu$-calibrable set suddenly vanishes, and that every suddenly vanishing set is $\mu$-Cheeger in itself. In the convex case, those implications are in fact equivalences, but one should be careful that in general at the critical point (when $\alpha=\lambda_{F}$ ) the set of solutions strictly contains $(\{\emptyset, F\})$, since uniqueness may not hold in the generalized Cheeger problem.

Acknowledgements. We would like to thank Luigi Ambrosio and Corinne Vachier for fruitful discussions about this work. V. Duval and J.-F. Aujol acknowledge support by the French "Agence Nationale de la Recherche" (ANR), under grant NATIMAGES (ANR-08-EMER-009) "Adaptivity for natural images and texture representations". This work has been supported by the grant FREEDOM (ANR07-JCJC-0048-01), "Movies, restoration and missing data".

Appendix A. We know state a few lemmas that are needed in the proof of Theorem 7.9. For convenience, we shall write $w(x):=h(x) \nu(x)$ (we have $w \in C_{c}^{1}\left(\mathbb{R}^{2}, \mathbb{R}^{2}\right)$, with $\operatorname{supp} w \subset \subset V)$.

LEMMA 7.13. Under the hypothesis of Theorem 7.9:

$$
\lim _{\alpha \rightarrow 0^{+}} \int_{U} \lambda(x)\left(\frac{\operatorname{det}(I+\alpha D(h \nu))-1}{\alpha}\right)=\int_{U} \lambda \operatorname{Tr}(D(h \nu)) h
$$

Proof. Recall that when $A$ and $H$ are two matrices:

$$
\lim _{t \rightarrow 0} \frac{\operatorname{det}(A+t H)-\operatorname{det} A}{t}=(\operatorname{det} A) \operatorname{Tr}\left(A^{-1} H\right)
$$

Therefore $\frac{d}{d \alpha} \operatorname{det}(I+\alpha D w)=\operatorname{det}(I+\alpha D w) \operatorname{Tr}\left((I+\alpha D w)^{-1} D w\right)$, and by the mean value theorem:

$$
\frac{\operatorname{det}(I+\alpha D w)-1}{\alpha} \leq \sup _{\beta \in[0, \alpha]} \operatorname{det}(I+\beta D w)\left(\operatorname{Tr}(I+\beta D w)^{-1} D w\right)
$$

which is uniformly bounded on $U$.

As a consequence, the dominated convergence theorem applies, and the result follows. $\square$

LEMMA 7.14. Under the hypothesis of Theorem 7.9:

$$
\lim _{\alpha \rightarrow 0^{+}} \int_{U} \frac{\lambda(x+\alpha \nu h)-\lambda(x)}{\alpha} \operatorname{det}(I+\alpha D w) d x=\int_{U}(D \lambda, h \nu)
$$




\section{Proof.}

Let $U_{1}, U_{2}$ be two open sets, such that $U_{2} \subset \subset U_{1} \subset \subset U$ and $|D \lambda|\left(U \backslash U_{2}\right) \leq \epsilon$ (which is possible since $U$ is open). Let us choose $\varphi \in C_{c}^{1}, 0 \leq \varphi \leq 1$, such that $\varphi(x)=1$ for $x \in U_{1}, \operatorname{supp} \varphi \subset U$, and $\left|\langle(D \lambda, w), \varphi\rangle-\int_{U}(D \lambda, w)\right| \leq \epsilon$. Notice that for $\alpha$ small enough, $\psi_{\alpha}\left(U \backslash U_{1}\right) \subset U \backslash U_{2}$, and we can even assume that $1 / 2 \leq \operatorname{det} D \psi_{\alpha} \leq 2$ uniformly on $U$.

The result will follow from the following inequality:

$$
\begin{aligned}
\left|\int_{U} \frac{\lambda(x+\alpha w)-\lambda(x)}{\alpha} \operatorname{det} D \psi_{\alpha} d x-\int_{U}(D \lambda, w)\right| \leq & \left|\int_{U} \frac{\lambda(x+\alpha w)-\lambda(x)}{\alpha}\left(\operatorname{det} D \psi_{\alpha}-\varphi\right) d x\right| \\
& +\left|\int_{U} \frac{\lambda(x+\alpha w)-\lambda(x)}{\alpha} \varphi d x-\int_{U}(D \lambda, w)\right|
\end{aligned}
$$

- Let us show that:

$$
\left|\int_{U} \frac{\lambda(x+\alpha w)-\lambda(x)}{\alpha}\left(\operatorname{det} D \psi_{\alpha}-\varphi\right) d x\right| \leq 6|D \lambda|\left(U \backslash U_{2}\right)\|w\|_{\infty}
$$

Since $\varphi \equiv \operatorname{det} D \psi_{\alpha} \equiv 1$ on $U_{1}$, it suffices to prove that:

$$
\int_{U \backslash U_{1}}\left|\frac{\lambda(x+\alpha w)-\lambda(x)}{\alpha}\right| d x \leq 2|D \lambda|\left(U \backslash U_{2}\right)\|w\|_{\infty}
$$

We proceed by approximation: let $\lambda_{n} \in C^{\infty}(U)$ converging to $\lambda$ in $L^{1}$ and $\left|D \lambda_{n}\right|\left(U \backslash U_{2}\right) \rightarrow|D \lambda|\left(U \backslash U_{2}\right)$.

$$
\begin{aligned}
\int_{U \backslash U_{1}}\left|\lambda_{n}(x+\alpha w)-\lambda_{n}(x)\right| d x & \leq \alpha \int_{U \backslash U_{1}} \int_{0}^{1}\left|\left\langle\nabla \lambda_{n}(x+\alpha w s), w\right\rangle\right| d s d x \\
& \leq \alpha \int_{U \backslash U_{1}} \int_{0}^{1}\left|\nabla \lambda_{n}(x+\alpha w s)\right| d s d x\|w\|_{\infty} \\
& \leq \alpha \int_{0}^{1} \int_{U \backslash U_{1}}\left|\nabla \lambda_{n}(x+\alpha w s)\right| d s d x\|w\|_{\infty} \\
& \leq \alpha \int_{0}^{1} \int_{U \backslash U_{2}}\left|\nabla \lambda_{n}((y))\right| \operatorname{det} D \psi_{s \alpha}^{-1} d y\|w\|_{\infty} \text { since } \psi_{s \alpha}\left(U \backslash U_{1}\right) \subset U \backslash U_{2} \\
& \leq 2 \alpha\left\|\nabla \lambda_{n}\right\|_{L^{1}\left(U \backslash U_{2}\right)}\|w\|_{\infty}=\alpha\left|D \lambda_{n}\right|\left(U \backslash U_{2}\right)\|w\|_{\infty}
\end{aligned}
$$

We pass to the limit $n \rightarrow+\infty$ to get the inequality.

- On the other hand, we have:

$$
\int_{U} \frac{\lambda(x+\alpha w(x))-\lambda(x)}{\alpha} \varphi(x) d x=\frac{1}{\alpha} \int_{U_{\alpha}} \lambda(y) \varphi\left(\psi_{\alpha}^{-1}(y)\right)\left|\operatorname{det} D \psi_{\alpha}^{-1}\right| d y-\frac{1}{\alpha} \int_{U} \lambda(x) \varphi(x) d x
$$

But for $y \in U \backslash U_{\alpha}, \psi_{\alpha}^{-1}(y) \notin U$ thus $\varphi\left(\psi_{\alpha}^{-1}(y)\right)=0$. Therefore: 


$$
\begin{aligned}
& \int_{U} \frac{\lambda(x+\alpha w(x)-\lambda(x))}{\alpha} \varphi(x) d x=\int_{U} \lambda(y)(\underbrace{\frac{\varphi\left(\psi_{\alpha}^{-1}(y)\right)-\varphi(y)}{\alpha}}_{\rightarrow-\nabla \varphi \cdot w})|\underbrace{\operatorname{det} D \psi_{\alpha}^{-1}}_{\rightarrow 1}| d y \\
&+\int_{U} \lambda(y) \varphi(y) \underbrace{\frac{\operatorname{det} D \psi_{\alpha}^{-1}-1}{\alpha}}_{\rightarrow-\operatorname{Tr} D w} d y
\end{aligned}
$$

The quotients are uniformly bounded (by the mean value theorem) and converge pointwise. Since $\lambda \in L^{1}$, we can apply Lebesgue's dominated convergence theorem, which gives us the following limit:

$$
-\int_{U} \lambda(y) \nabla \varphi(y) \cdot w-\int_{U} \lambda \varphi \operatorname{div} w=\langle(D \lambda, w), \varphi\rangle
$$

- Finally:

$$
\begin{aligned}
\limsup _{\alpha \rightarrow 0}\left|\int_{U} \frac{\lambda(x+\alpha w)-\lambda(x)}{\alpha} \operatorname{det} D \psi_{\alpha} d x-\int_{U}(D \lambda, w)\right| & \leq \epsilon+6|D \lambda|\left(U \backslash U_{2}\right)\|w\|_{\infty} \\
& \leq\left(1+6\|w\|_{\infty}\right) \epsilon
\end{aligned}
$$

口

and this is true for every $\epsilon>0$, hence the result.

\section{Appendix B.}

Proposition 7.15. Let $F \subset \mathbb{R}^{2}$ be a $\mu$-calibrable set. Then:

(i)

$$
\frac{\text { Per } F}{\mu(F)} \leq \frac{\text { Per } U}{\mu(U \cap F)} \text { for all } U \subset \mathbb{R}^{2}
$$

(ii) Each connected component of $F$ is convex.

Proof. We have:

$\mu(U \cap F)=\int_{\mathbb{R}^{2}} \chi_{U} \chi_{F} d \mu=\frac{1}{\lambda_{F}} \int_{\mathbb{R}^{2}} \chi_{U}(-\operatorname{div} \xi) d x=\frac{1}{\lambda_{F}} \int_{\mathbb{R}^{2}}\left(\xi, D \chi_{U}\right) \leq\|\xi\|_{\infty} \operatorname{Per} U \frac{\mu(F)}{\operatorname{Per} F}$

口

Proposition 7.16. Let $C \subset \mathbb{R}^{2}$ be a bounded convex set with finite perimeter. The following assertions are equivalent:

(i) $C$ is $\mu$-calibrable

(ii) $C$ is a solution of the problem:

$$
\min _{X \subset C} \operatorname{Per} X-\lambda_{C} \mu(X) \quad\left(\text { where } \lambda_{C}=\frac{\operatorname{Per} C}{\mu(C)}\right)
$$

Proof. 
(i) $\Rightarrow$ (ii): Let $X$ be a set of finite perimeter. We have:

$$
\lambda_{C} \lambda(C \cap X)=\int_{\mathbb{R}^{2}} \lambda_{C} \chi_{C} \chi_{X} d \mu=-\int_{\mathbb{R}^{2}} \operatorname{div} z \chi_{X} d x=\int_{\mathbb{R}^{2}}\left(z, D \chi_{X}\right) \leq \operatorname{Per} X
$$

Thus Per $X-\lambda_{C} \mu(X) \geq 0=\operatorname{Per} C-\lambda_{C} \mu(C)$.

(ii) $\Rightarrow$ (i): Let $w \in L_{\lambda}^{1} \cap B V$. Up to a decomposition of $w$ in $w^{+}-w^{-}$, and since $|w|_{T V}=\left|w^{+}\right|_{T V}+\left|w^{-}\right|_{T V}$ by the coarea formula, we can assume that $w$ is non-negative. Then, writing $f=\lambda_{C} \chi_{C}$ :

$$
\begin{aligned}
\int f w d \mu & =\int_{0}^{+\infty} \int_{\mathbb{R}^{2}} \lambda_{C} \chi_{C} \chi_{\{w \geq t\}} d \mu d t \\
& =\int_{0}^{+\infty} \lambda_{C} \mu(C \cap\{w \geq t\} d t \\
& \leq \int_{0}^{+\infty} \operatorname{Per}(C \cap\{w \geq t\} d t \text { since } C \text { is minimal } \\
& \leq \int_{0}^{+\infty} \operatorname{Per}(\{w \geq t\} d t \text { since } C \text { is convex } \\
& \leq|w|_{T V}
\end{aligned}
$$

Therefore we have proved that $\left\|\lambda_{C} \chi_{C}\right\|_{*, \lambda} \leq 1$. Consequently, there is some $z \in X_{\infty}$, such that $-\operatorname{div} z=\lambda_{C} \chi_{C} \lambda$.

口

\section{REFERENCES}

[1] W.K. Allard, Total variation regularization for image denoising II: Examples. Accepted (2008).

[2] - Total variation regularization for image denoising I: Geometric theory, SIAM Journal on Mathematical Analysis, 39 (2007).

[3] S. AlLiney, Digital filters as absolute norm regularizers, IEEE Transactions on Signal Processing, 40 (1992), pp. 1548-1562.

[4] - Recursive median filters of increasing order : a variational approach, IEEE Transactions on Signal Processing, 44 (1996), pp. 1346-1354.

[5] F. Alter, Variation totale : théorie et applications, PhD thesis, ENS Cachan, janvier 2008.

[6] F. Alter, V. Caselles, and A. Chambolle, A characterization of convex calibrable sets in $\mathbb{R}^{n}$, Math. Ann., 7 (2005), pp. 29-53.

[7] - Evolution of convex sets in the plane by minimizing the total variation flow, Interfaces and Free Boundaries, 332 (2005), pp. 329-366.

[8] L. Ambrosio, Minimizing movements.

[9] Corso introduttivo alla teoria geometrica della misura ed alle superfici minimime, Quad. Scuola Norm. Sup. Pisa, Pantograph, Genova, 1997.

[10] L. Ambrosio, N. Fusco, And D. Pallara, Functions of Bounded Variation and Free Discontinuity Problems, Oxford mathematical monographs, Oxford University Press, 2000.

[11] F. Andreu-Vaillo, V. Caselles, and J. M. Mazon, Parabolic quasilinear equations minimizing linear growth functionals, vol. 223 of Progress in Mathematics, Birkhauser, 2002.

[12] G. Anzellotti, Pairing between measures and bounded functions and compensated compactness, Ann. di Matematica Pura ed Appl., IV (1983), pp. 293-318.

[13] J-F. Aujol, G. Aubert, L. Blanc-Féraud, and A. Chambolle, Image decomposition into a bounded variation component and an oscillating component, Journal of Mathematical Imaging and Vision, 22 (2005), pp. 71-88.

[14] J-F. Aujol and A. Chambolle, Dual norms and image decomposition models, International Journal of Computer Vision, 63 (2005), pp. 85-104. 
[15] J-F. Aujol, G. Gilboa, T. Chan, And S. Osher, Structure-texture image decomposition modeling, algorithms, and parameter selection, International Journal of Computer Vision, 67 (2006), pp. 111-136.

[16] E. Barozzi, E. Gonzales, And I. Tamanini, The mean curvature of a set of finite perimeter, AMS, 99 (1987), pp. 313-316.

[17] G. Bellettini, V. Caselles, A. Chambolle, and M. Novaga, The volume preserving crystalline mean curvature flow of convex sets in $\mathbf{r}^{n} .2007$.

[18] G. Bellettini, V. Caselles, and M. Novaga, The total variation flow in $r^{n}$, J. Differential Equations, 184 (2002), pp. 475-525.

[19] G. Bellettini, M. Novaga, And M. Paolini, Characterization of facet-breaking for nonsmooth mean curvature flow in the convex case, Interfaces and Free Boundaries, 3 (2001), pp. $415-446$.

[20] G. Buttazzo, G. Carlier, and M. Comte, On the selection of maximal cheeger sets, Differential and Integral Equations, 20 (2007), pp. 991-1004.

[21] G. Carlier And M. Comte, On a weighted total variation minimization problem, J. Funct. Anal., 250 (2007), pp. 214-226.

[22] G. Carlier, M. Comte, and G. Peyré, Approximation of maximal cheeger sets by projection. 2008.

[23] V. Caselles, A. Chambolle, S. Moll, and M. Novaga, A characterization of convex calibrable sets in $\mathbb{R}^{n}$ with respect to anisotropic norms. 2005.

[24] V. Caselles, A. Chambolle, And M. Novaga, Uniqueness of the cheeger set of a convex body. 2006.

[25] T.F. Chan AND S. Esedoglu, Aspects of total variation regularized $L^{1}$ function approximation, SIAM Journal on Applied Mathematics, 65 (2005), pp. 1817-1837.

[26] J. DARBON, Total variation minimization with $L^{1}$ data fidelity as a contrast invariant filter, 4th International Symposium on Image and Signal Processing and Analysis (ISPA 2005), (2005), pp. 221-226.

[27] J. DARbon AND M. Sigelle, Image restoration with discrete constrained total variation part I: Fast and exact optimization, Journal of Mathematical Imaging and Vision, 26 (2006), pp. 261-276.

[28] F. Dibos AND G. Koepfler, Total variation minimization by the fast level sets transform, IEEE Workshop Variational and Level Set Methods in Computer Vision., (2001), pp. 179185.

[29] E. GiUsti, On the equation of surfaces of prescribed mean curvature, Invent. Math., 46 (1978), pp. $111-137$.

[30] F. Guichard, J.-M. Morel, And R. Ryan, Contrast invariant image analysis and PDE's. preprint.

[31] B. Kawohl And T. LACHAND-RoBert, Characterization of cheeger sets for convex subsets of the plane, Pacific J. Math., 225 (2006), pp. 103-118.

[32] G. Matheron, Random Sets and Integral Geometry, John Wiley \& Sons, New York, 1975.

[33] Y. MEYER, Oscillating patterns in image processing and nonlinear evolution equations, vol. 22 of University Lecture Series, American Mathematical Society, Providence, RI, 2001. The fifteenth Dean Jacqueline B. Lewis memorial lectures.

[34] P. Monasse And F. Guichard, Scale-space from a level lines tree, Journal of Visual Communication and Image Representation, 11 (2000), pp. $224-236$.

[35] M. Nikolova, A variational approach to remove outliers and impulse noise, JMIV, 20 (2004), pp. $99-120$.

[36] S.J. Osher, A. SOle, AND L.A. Vese, Image decomposition and restoration using total variation minimization and the $H^{-1}$ norm, Multiscale Modeling and Simulation: A SIAM Interdisciplinary Journal, 1 (2003), pp. 349-370.

[37] T. Rockafellar, Convex Analysis, vol. 224 of Grundlehren der mathematischen Wissenschaften, Princeton University Press, second ed., 1983.

[38] L. Rudin, S. Osher, And E. FAtemi, Nonlinear total variation based noise removal algorithms, Physica D, 60 (1992), pp. 259-268.

[39] P. Salembier, A. Oliveras, and L. Garrido, Anti-extensive connected operators for image and sequence processing, IEEE Transactions on Image Processing, 7 (1998), pp. 555-570.

[40] P. Sollle, Morphological Image Analysis: Principles and Applications, Springer-Verlag New York, Inc., Secaucus, NJ, USA, 2003.

[41] L.A. VESE AND S.J. Osher, Modeling textures with total variation minimization and oscillating patterns in image processing, Journal of Scientific Computing, 19 (2003), pp. 553-572.

[42] K.R. Vixie And S. Esedoglu, Some properties of minimizers for the Chan-Esedoglu $L^{1} T V$ functional, (2007). 
[43] W. Yin, D. Goldfarb, And S. Osher, A comparison of three total variation based texture extraction models, Journal of Visual Communication and Image Representation, 18 (2007), pp. 240-252.

[44] W. YIn, W. GOLDFARB, AND S. OShER, The total variation regularized $L^{1}$ model for multiscale decomposition, SIAM Journal on Multiscale Modeling and Simulation, 6 (2007), pp. 190211. 